\title{
Baicalin Ameliorates Cognitive Impairment and Protects Microglia from LPS-Induced Neuroinflammation via the SIRT1/HMGB1 Pathway
}

\author{
Yue Li, ${ }^{1}$ Taotao Liu, ${ }^{1}$ Yitong $\mathrm{Li}^{1}{ }^{1}$ Dengyang Han, ${ }^{1}$ Jingshu Hong, ${ }^{1}$ Ning Yang, ${ }^{1}$ Jindan He, \\ Ronghui Peng, ${ }^{2}$ Xinning Mi, ${ }^{1}$ Chongshen Kuang, ${ }^{1}$ Yang Zhou, ${ }^{1}$ Yongzheng Han, ${ }^{1}$ \\ Chengmei Shi, ${ }^{1}$ Zhengqian $\mathrm{Li}\left(\mathbb{D},{ }^{1}\right.$ and Xiangyang Guo ${ }^{1}{ }^{1}$ \\ ${ }^{1}$ Department of Anesthesiology, Peking University Third Hospital, No. 49 North Garden Road, Haidian District, \\ Beijing 100191, China \\ ${ }^{2}$ Department of Anesthesiology, National Cancer Center/National Clinical Research Center for Cancer/Cancer Hospital, \\ Chinese Academy of Medical Sciences and Peking Union Medical College, No. 17 Pan-Jia-Yuan nanli Road, Chaoyang District, \\ Beijing 100021, China
}

Correspondence should be addressed to Zhengqian Li; zhengqianli@hsc.pku.edu.cn and Xiangyang Guo; puthmzk@hsc.pku.edu.cn Received 17 April 2020; Revised 20 August 2020; Accepted 4 September 2020; Published 22 September 2020

Academic Editor: L szl Vir g

Copyright (c) 2020 Yue Li et al. This is an open access article distributed under the Creative Commons Attribution License, which permits unrestricted use, distribution, and reproduction in any medium, provided the original work is properly cited.

\begin{abstract}
Systemic inflammation often induces neuroinflammation and disrupts neural functions, ultimately causing cognitive impairment. Furthermore, neuronal inflammation is the key cause of many neurological conditions. It is particularly important to develop effective neuroprotectants to prevent and control inflammatory brain diseases. Baicalin (BAI) has a wide variety of potent neuroprotective and cognitive enhancement properties in various models of neuronal injury through antioxidation, antiinflammation, anti-apoptosis, and stimulating neurogenesis. Nevertheless, it remains unclear whether BAI can resolve neuroinflammation and cognitive decline triggered by systemic or distant inflammatory processes. In the present study, intraperitoneal lipopolysaccharide (LPS) administration was used to establish neuroinflammation to evaluate the potential neuroprotective and anti-inflammatory effects of BAI. Here, we report that BAI activated silent information regulator 1 (SIRT1) to deacetylate high-mobility group box 1 (HMGB1) protein in response to acute LPS-induced neuroinflammation and cognitive deficits. Furthermore, we demonstrated the anti-inflammatory and cognitive enhancement effects and the underlying molecular mechanisms of BAI in modulating microglial activation and systemic cytokine production, including tumor necrosis factor(TNF-) $\alpha$ and interleukin- (IL-) $1 \beta$, after LPS exposure in mice and in the microglial cell line, BV2. In the hippocampus, BAI not only reduced reactive microglia and inflammatory cytokine production but also modulated SIRT1/HMGB1 signaling in microglia. Interestingly, pretreatment with SIRT1 inhibitor EX-527 abolished the beneficial effects of BAI against LPS exposure. Specifically, BAI treatment inhibited HMGB1 release via the SIRT1/HMGB1 pathway and reduced the nuclear translocation of HMGB1 in LPS-induced BV2 cells. These effects were reversed in BV2 cells by silencing endogenous SIRT1. Taken together, these findings indicated that BAI reduced microglia-associated neuroinflammation and improved acute neurocognitive deficits in LPS-induced mice via SIRT1-dependent downregulation of HMGB1, suggesting a possible novel protection against acute neurobehavioral deficits, such as delayed neurocognitive recovery after anesthesia and surgery challenges.
\end{abstract}

\section{Introduction}

Delayed neurocognitive recovery (dNCR), formally known as postoperative cognitive decline (POCD) [1], is characterized by a decline in cognitive function that occurs in patients up to
30 days after anesthesia and surgery. The development of dNCR has been reported to be associated with daily function impairment and increased morbidity and mortality leading to an economic burden on healthcare resources [2]. Previous studies including ours have found that neuroinflammation 
has a key role in the pathophysiology of dNCR $[3,4]$. Bloodborne factors, as well as the proinflammatory systemic milieu, can adversely affect the central nervous system (CNS) function by directly affecting synaptic plasticity and cognitive function [5]. Systemic alarmins and cytokines such as interleukin- (IL-) $1 \beta$, tumor necrosis factor- (TNF-) $\alpha$, and high-mobility group box 1 (HMGB1) protein trigger neuroinflammation after peripheral surgery in mouse models [6]. Activated microglia, which secrete proinflammatory factors, have been well documented to play an important role in CNS dyshomeostasis. Lipopolysaccharide (LPS) can trigger a systemic inflammatory response via activation of pattern recognition receptors (including toll-like receptors), cytokines, and oxidative stress pathways. We chose the intraperitoneal injection of LPS, which is a more clinical method, rather than intracranial LPS injection. This method is an effective animal model for dNCR $[7,8]$.

Silent information regulator 1 (SIRT1) is an NADdependent nuclear histone deacetylase that plays important roles in maintaining cellular homeostasis including metabolism, cell survival, and insulin resistance [9]. SIRT1 also plays a significant role in regulating normal brain functions such as plasticity and memory $[10,11]$. Furthermore, it has been demonstrated that SIRT1 deacetylates inflammation-related transcription factors such as NF- $\kappa \mathrm{B}$ to reverse inflammation [12]. Decreased expression levels of SIRT1 have been observed in the hippocampus of aged dNCR model mice [13]. dNCR model mice respond to therapies by upregulating SIRT1 expression in the hippocampus, as shown by a neuroprotective treatment of aged mice with SIRT1 activators [14, 15]. Recent reports have shown that SIRT1 is involved in protein quality control, as SIRT1 suppressed aggregation of tau proteins [16] and HMGB1 transcription [17] by direct deacetylation regulation.

HMGB1, a damage-associated molecular pattern (DAMP) family member, is a ubiquitous nuclear protein that contributes to the chromatin architecture in physiological conditions [18]. Under pathophysiological conditions, HMGB1 acts as a pathogenic inflammatory factor. Its translocation from the nucleus to the extracellular space is mediated by acetylation; it is released by glia and neurons upon inflammasome activation via an $\mathrm{N}$-methyl $\mathrm{D}$-aspartate receptor subtype 2B- (NR2B-) mediated mechanism [19]. Extracellular HMGB1 mediates sterile inflammation and induces cells to release inflammatory cytokines by binding to receptor for advanced glycation end products (RAGE) and toll-like receptor 4 (TLR4). This binding then activates the NF- $\kappa$ B pathway directly or indirectly via the phosphoinositide 3-kinase (PI3K) or mitogen-activated protein kinases (MAPKs) pathway to promote inflammation and facilitate the upregulation of HMGB1 [20]. Moreover, previous reports have demonstrated that HMGB1 serves as an adverse factor in memory impairment, neurodegeneration, and neuroinflammatory progression.

Baicalin (BAI) is an important flavonoid compound, which is responsible for most of the biological effects of Scutellaria baicalensis [21]. BAI is a Food and Drug Administration- (FDA-) approved pharmaceutical food flavonoid. BAI could penetrate the blood-brain barrier (BBB) and distribute into the cerebrospinal fluid, which tends to accumulate in the striatum, thalamus, and hippocampus [22]. Additionally, BAI administration increased the expression of $\mathrm{GABA}_{\mathrm{A}}$ receptor and $\alpha$-amino-3-hydroxy-5-methyl-4-isoxazolepropionic acid (AMPA) receptor and decreased the activation of NLRP3 inflammasome in the hippocampus [21]. A number of studies have shown that BAI ameliorates various inflammatory diseases and prevents neurodegenerative diseases through various mechanisms [23-26], including suppression of oxidative stress, apoptosis, and inflammation, neurogenesis stimulation, and promotion of brain-derived neurotrophic factor (BDNF) expression. Moreover, the neuroprotective effects of BAI in neurological disorders like depressive-like behaviors [12] or oxidative stress injury of SH-SY5Y cells [27] have been linked to SIRT1 activation. BAI dramatically suppressed the HMGB1 protein expression in the hippocampal tissues of chronic unpredictable mild stress- (CUMS-) induced mice [28]. BAI also mitigates long-term LPS-evoked depression model symptoms by alleviating microglial activation $[29,30]$. To date, no studies have elucidated whether BAI affects the acute LPS-induced microglial activation and inflammatory response in a SIRT1dependent manner. In the current study, we explored the cognitive-improving effects of BAI upon single administration of LPS induction. We also investigated the underlying neuroprotective mechanisms with emphasis on improving microglial activation and neuroinflammation through SIRT1 upregulation and repression of the HMGB1 signaling pathway, thereby suggesting a new role of BAI in mediating cognition decline.

\section{Materials and Methods}

2.1. Drugs and Reagents. BAI (Figure 1(a); 98\% purity, CAS number 21967-41-9) was bought from Tauto Biotech (Shanghai, China). LPS from Escherichia coli O111:B4 and protease and phosphatase inhibitor tablets were purchased from Sigma-Aldrich (St. Louis, MO, USA). EX-527, also known as Selisistat, was purchased from Selleck (Houston, TX, USA). Rabbit anti-SIRT1, rabbit anti-HMGB1, rabbit antiacetylated lysine, and mouse anti-GFAP were from Cell Signaling Technology (Beverly, MA, USA); mouse antiTNF- $\alpha$ and mouse anti-HMGB1 were from Santa Cruz Biotechnology (Santa Cruz, CA); rabbit anti-IL-1 $\beta$, goat antimouse IgG H\&L (Alexa Fluor ${ }^{\circledR} 594$ ), and goat antirabbit IgG H\&L (Alexa Fluor ${ }^{\circledR}$ 594/488) were from Abcam (Cambridge, MA, USA); rabbit anti-Iba-1 was from Wako (Rosemont, IL, USA); mouse anti-LaminB1, mouse anti-GAPDH, and mouse anti- $\beta$-actin were from Proteintech Group (Wuhan, China). Protein A/G-coupled magnetic beads were from Invitrogen (Carlsbad, CA, USA).

2.2. Animals. Adult male C57BL/6 mice (10-11weeks, 21$23 \mathrm{~g}$ ) were purchased from the Department of Laboratory Animal Research Center, Peking University, and maintained under standard laboratory conditions. All mice were acclimated for 1 week in a controlled environment of $24 \pm 1^{\circ} \mathrm{C}$, $60 \%$ humidity, and 12:12 h light-dark cycle with free access to food and water. All animal experiments were conducted 
<smiles>O=C(O)C1OC(Oc2cc3oc(-c4ccccc4)cc(=O)c3c(O)c2O)[C@H](O)[C@H](O)[C@@H]1O</smiles>

(a)

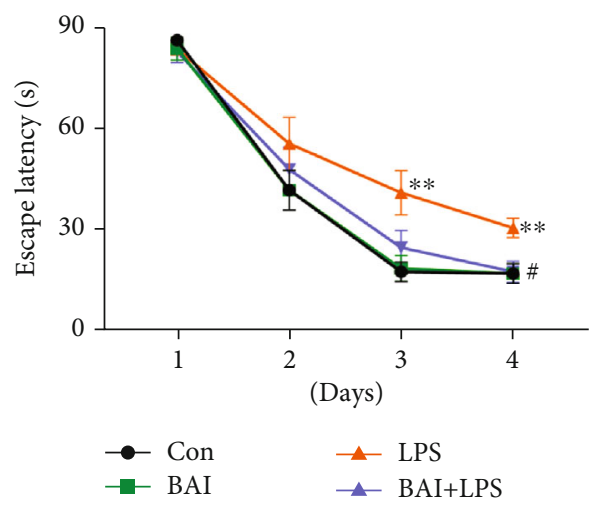

(c)

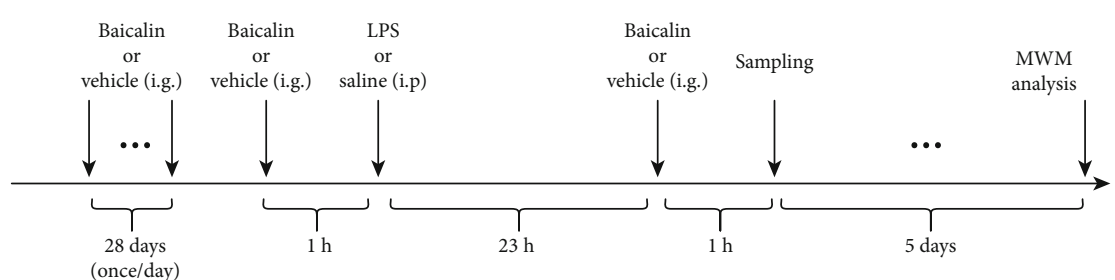

(b)

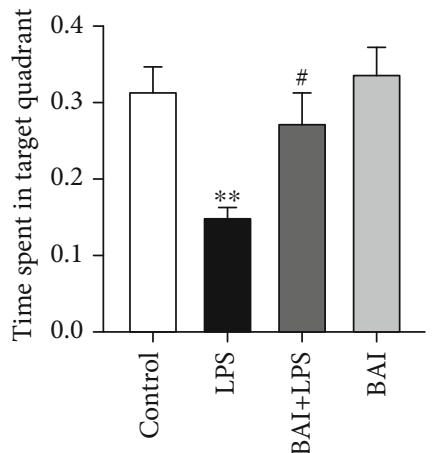

(d)

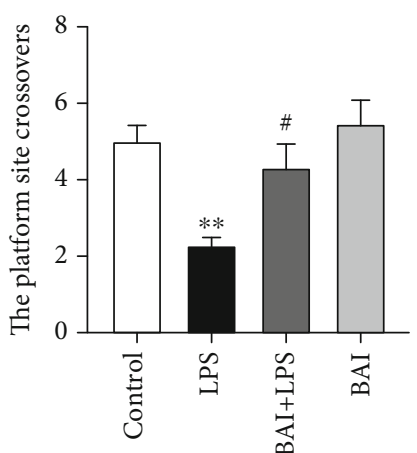

(e)

FIGURE 1: BAI ameliorated learning and memory deficits in LPS-induced mice. Mice were divided into four groups: control, LPS, BAI+LPS, and BAI. (a) Chemical molecular formula of BAI. (b) Schematic timeline of the experimental procedure. (c) The escape latency of LPSinduced mice in the MWM navigation test. (d) Analysis of the time spent in each quadrant during the probe trial of the MWM. (e) The platform crossing times during the probe trial of the MWM test. Data are shown as means \pm SEM $(n=10-12$ per group) and were compared by repeated measure 2-way ANOVA with Bonferroni post hoc analysis. ${ }^{*} P<0.05,{ }^{* *} P<0.01$, control vs. LPS; ${ }^{*} P<0.05$, BAI +LPS vs. LPS. BAI, baicalin; MWM, Morris water maze; LPS, lipopolysaccharide; SEM, standard error of the mean.

in accordance with the guidelines for experimental animal use, and the protocol was approved by the animal ethics committee of Peking University Health Science Center.

2.3. Experimental Model and Drug Administration. After the acclimation, mice were randomly assigned into six groups ( $n=8-12$ in each group): (i) control $(0.9 \% \mathrm{NaCl}$, intraperitoneal injection i.p.), (ii) LPS (2 mg/kg i.p.), (iii) LPS+BAI $(2 \mathrm{mg} / \mathrm{kg} \mathrm{LPS} \mathrm{i.p.+103} \mathrm{mg/kg} \mathrm{BAI,} \mathrm{intragastric} \mathrm{gavage} \mathrm{i.g.,}$ daily), (iv) BAI alone (103 mg/kg), (v) BAI+LPS+EX-527 (a selective SIRT1 inhibitor, $5 \mathrm{mg} / \mathrm{kg} /$ day, every 2 days for a total of 7 days before LPS and $5 \mathrm{mg} / \mathrm{kg} /$ day after LPS), and (vi) LPS+EX-527. We chose this LPS dose based on previous studies in which LPS at $2 \mathrm{mg} / \mathrm{kg}$ reliably induced sickness behavior and learning deficits in C57BL/6 J mice [31, 32]. The chemical structure of BAI is shown in Figure 1(a). BAI, suspended in water containing $0.3 \%$ carboxymethylcellulose sodium (CMC-Na), was administered daily by intragastric gavage, which was started 4 weeks before LPS treatment and continued for another 6 days for a total of 34 days (Figure 1(b)). The behavioral tests were performed $24 \mathrm{~h}$ after the LPS administration. The doses of BAI and EX-527 were chosen based on earlier reports in which BAI significantly improved learning and memory deficits of APP/PS1 mice and inhibited the expression of SIRT1, respectively $[33,34]$.

2.4. Morris Water Maze (MWM). The MWM, which is a hippocampus-dependent spatial navigation and reference memory test for rodents, was conducted as described previously with minor modifications [35]. The MWM (Sunny Instruments Co. Ltd., Beijing, China) was performed in a white circular tank ( $120 \mathrm{~cm}$ in diameter and $50 \mathrm{~cm}$ in height) filled with white water maintained at $23 \pm 1^{\circ} \mathrm{C}$. The maze was divided into four quadrants. An invisible fixed platform ( $10 \mathrm{~cm}$ in diameter) was immersed $1 \mathrm{~cm}$ below the water surface in the target quadrant. Mice were gently placed in one quadrant facing the wall of the MWM pool. The mice were allowed to swim for $90 \mathrm{~s}$ to locate the hidden platform during each trial. When successful, the mouse was allowed to stay for $10 \mathrm{~s}$ on the platform. If unsuccessful within the time limit, the mouse was guided to the platform for $10 \mathrm{~s}$. A 3-5 min interval was allowed between each trial. The time spent to locate the platform, swimming speed, and distance were recorded by a video camera. Four spatial acquisition trials were performed with each mouse per day. Latency (s) was measured to assess the time taken to reach the hidden platform for four consecutive days. On the following day, we performed the probe test for the evaluation of memory consolidation by removing the platform and allowing the animal to swim freely for $90 \mathrm{~s}$. The number of crossings over the previously hidden platform and the time spent in the specific target quadrant were recorded.

2.5. Immunohistochemistry and Immunofluorescence. Mice were transcardially perfused with phosphate-buffered saline (PBS, pH 7.3), followed by $50 \mathrm{ml}$ of $4 \%$ paraformaldehyde in PBS. Tissues were processed for paraffin embedding and 
6- $\mu \mathrm{M}$ sections were prepared. The paraffin sections were deparaffinized, rehydrated, and blocked. For immunohistochemistry, the sections were incubated with anti-Iba-1 $(1: 500)$ and anti-GFAP $(1: 200)$ overnight at $4^{\circ} \mathrm{C}$. Subsequently, the sections were washed with PBS and incubated with goat antirabbit or antimouse secondary antibodies at room temperature for $30 \mathrm{~min}$. Next, the 3, 3diaminobenzidine color reaction was performed. For immunofluorescence, the sections were incubated with antibodies against HMGB1, Iba-1, and SIRT1. Subsequently, the sections were incubated with Alexa Fluor ${ }^{\circledR} 594$ or Alexa Fluor ${ }^{\circledR}$ 488 secondary antibodies for $1 \mathrm{~h}$. The slides were imaged with Leica microsystems (Wetzlar, Germany). Immunohistochemical sections were evaluated by assessing the signal intensity (five grades) [20]: 0, no detectable stained cells; 1 , very low staining of cells; 2 , moderate density of positive cells; 3 , submaximal density of positive cells; and 4 , the highest density of positive cells.

2.6. Cell Culture. Murine BV2 microglial cells were purchased from China Infrastructure of Cell Line Resource (Beijing, China) and cultured in Dulbecco's modified Eagle medium (DMEM) (Gibco, Invitrogen) supplemented with 10\% fetal bovine serum (FBS) (Gibco, Invitrogen) at $37^{\circ} \mathrm{C}$ in a humidified incubator chamber under an atmosphere of $5 \% \mathrm{CO}_{2}$. The cells were pretreated with BAI $(40 \mu \mathrm{M})$ for $2 \mathrm{~h}$ followed by incubation with LPS $(100 \mathrm{ng} / \mathrm{ml})$ for $24 \mathrm{~h}$.

For small interfering RNA (siRNA) transfection, microglia were transfected with SIRT1-specific siRNA (GenePharma, Shanghai, China) or negative control siRNA for $6 \mathrm{~h}$ using RNAiMAX (Invitrogen) with Opti-MEM Reduced-Serum Medium (Gibco, Invitrogen). Then, cells were maintained in DMEM supplemented with $10 \%$ FBS at $37^{\circ} \mathrm{C}$ in a humidified incubator chamber under an atmosphere of $5 \% \mathrm{CO}_{2}$ for an additional $36 \mathrm{~h}$. Next, the microglia were exposed to LPS $(100 \mathrm{ng} / \mathrm{ml})$ with or without BAI $(40 \mu \mathrm{M})$. Western blot analysis was used to evaluate the siRNA efficiency.

2.7. Cell Viability. The Cell Counting Kit-8 (CCK8, Dojindo, Kumamoto, Japan) was used to assess the viability of BV2 cells. Cells were incubated in DMEM+10\% FBS in 96-well plates at a density of $1 \times 10^{4}$ cells/well in a $5 \% \mathrm{CO}_{2}$ incubator and treated with various concentrations of BAI for $24 \mathrm{~h}$. Thereafter, $10 \mu \mathrm{l}$ of CCK8 reagent was added, and the plates were incubated at $37^{\circ} \mathrm{C}$ for $2 \mathrm{~h}$ in a $5 \% \mathrm{CO}_{2}$ incubator. Absorbance was measured at $450 \mathrm{~nm}$.

2.8. Determination of Extracellular HMGB1 Levels. The HMGB1 levels released into the culture media were determined based on previously published methods [36]. Equal aliquots of conditioned media from equal numbers of BV2 cells that were treated with the indicated reagents for the indicated durations were used to measure the amount of HMGB1 secreted into the culture media. Eighty percent ice-cold acetone was used to precipitate the proteins in the conditioned media at $-20^{\circ} \mathrm{C}$ for $1 \mathrm{~h}$. After centrifugation at $16000 \times g$ for $10 \mathrm{~min}$ at $4^{\circ} \mathrm{C}$, the protein pellets were washed with $80 \%$ ice-cold acetone. The pellets were then resus- pended in SDS-PAGE sample buffer, and the levels of HMGB1 were determined by immunoblot analysis.

2.9. Western Blot Analysis. Hippocampal tissues of LPStreated mice and treated BV2 cells were lysed with RIPA buffer containing protease and phosphatase inhibitors. The extracted proteins were then centrifuged at $15000 \times g$ for $10 \mathrm{~min}$ at $4^{\circ} \mathrm{C}$, and the concentration of the supernatants was quantified by the bicinchoninic acid (BCA) protein kit according to the manufacturer's instructions (Beyotime, Shanghai, China). Equal amounts of protein were separated by $10 \%$ SDS-PAGE and transferred onto polyvinylidene difluoride (PVDF) membranes. After blocking with 5\% FBS in TBS-T (TBS containing 0.1\% Tween 20), the membranes were incubated at $4^{\circ} \mathrm{C}$ overnight with the following primary antibodies: anti-SIRT1 $(1: 1000)$, anti-HMGB1 $(1: 1000)$, anti-TNF- $\alpha \quad(1: 1000), \quad$ anti-IL- $1 \beta \quad(1: 1000)$, anti-Iba- 1 $(1: 1000)$, anti-GFAP $(1: 1000)$, anti-LaminB1 $(1: 2000)$, anti-GAPDH $(1: 5000)$, and anti- $\beta$-actin $(1: 5000)$. After incubation with secondary antibodies for $1 \mathrm{~h}$ at room temperature, the membranes were exposed to enhance chemiluminescence and then analyzed using the Gel-Pro analyzer software (Rockville, MD, USA).

2.10. Coimmunoprecipitation. Whole-cell lysates were precleared with protein A/G-coupled magnetic beads and then incubated with $1 \mathrm{mg}$ of anti-HMGB1 antibodies overnight at $4^{\circ} \mathrm{C}$. Subsequently, the mixture was incubated for $4 \mathrm{~h}$ with protein A/G magnetic beads. Samples were then extensively washed four times with PBS and then boiled. The immunoblot analysis was performed with antiacetylated lysine antibodies.

2.11. Immunocytochemistry. BV2 cells were washed with cold PBS and fixed with $4 \%$ paraformaldehyde solution for $10 \mathrm{~min}$ and then washed thrice with $1 \times$ PBS solution for $15 \mathrm{~min}$. Subsequently, the cells were permeabilized with $0.5 \%$ Triton X100 for $5 \mathrm{~min}$ and then blocked with PBS containing $10 \%$ BSA. The slides were incubated with anti-HMGB1 $(1: 100)$ overnight at $4^{\circ} \mathrm{C}$. After three washes, the cells were incubated with Alexa Fluor ${ }^{\circledR} 594$ secondary antibodies at $37^{\circ} \mathrm{C}$ for $1 \mathrm{~h}$ and the nuclei were stained with DAPI for $10 \mathrm{~min}$. Fluorescent images were obtained using a TCS SP8 X confocal fluorescence microscope (Mannheim, Germany).

2.12. Cytoplasmic and Nuclear Protein Extraction. Extraction was strictly performed according to the manufacturer's instructions for nuclear and cytoplasmic protein extraction kits (Beyotime Institute of Biotechnology, Shanghai, China). BV2 cells were lysed in cytosol fractionation buffer for $5 \mathrm{~min}$ and then centrifuged at $10000 \times \mathrm{g}$ at $4^{\circ} \mathrm{C}$ for $1 \mathrm{~min}$, to obtain the supernatant containing the cytosolic fraction. The pellet was lysed in nuclear fractionation buffer on ice for $15 \mathrm{~min}$. The nuclear fraction (supernatant) was obtained by centrifugation at $10000 \times \mathrm{g}$ at $4^{\circ} \mathrm{C}$ for $15 \mathrm{~min}$.

2.13. Statistical Analysis. Data from at least three experiments were used for analyses with the GraphPad Prism 8.0 Software. Quantitative data are shown as means \pm SEM. Oneway ANOVA followed by Bonferroni post hoc test was used 
for multiple comparisons. The MWM data were analyzed using two-way ANOVA with repeated measures followed by a Bonferroni post hoc test to analyze the difference in escape latency between each group. $P<0.05$ was considered significant.

\section{Results}

3.1. BAI Ameliorates LPS-Induced Spatial Learning and Memory Impairment. Previous studies have demonstrated that intraperitoneal administration of LPS leads to spatial learning and memory deficits $[7,31]$. Therefore, the protective effects of BAI on LPS-induced cognitive deficits were examined in this well-established mouse model. There were no significant differences in swimming speed among the four groups (data not shown). LPS-treated mice showed significantly enhanced escape latencies compared with the control mice on days 3 and 4 (Figure 1(c)), suggesting that LPS caused spatial learning impairments. Pretreating LPSstimulated mice with BAI significantly attenuated the escape latencies on day 4 of training (Figure 1(c)). There were no significant differences in latencies between the control and BAI groups. In the probe test, the frequency of crossing the platform and the time spent in the target quadrant were both significantly increased in the LPS-injected mice pretreated with BAI relative to the LPS group (Figures 1(d) and 1(e)), suggesting that memory impairments after LPS exposure can be remarkably alleviated by BAI administration. Collectively, these findings indicated that BAI treatment ameliorated LPS-induced spatial learning and memory impairments.

3.2. BAI Inhibits LPS-Stimulated Glial Activation and Cytokine Release in the Hippocampus. Evidence has shown that microglia and astrocytes are associated with proinflammatory responses upon surgical trauma. Inhibition of microglia can lead to a reduction in proinflammatory cytokines including TNF- $\alpha$ and IL- $1 \beta$, which are all important factors in neuroinflammation. Thus, we examined the effects of BAI on LPS-induced microglial and astrocyte activation in vivo. The LPS-injected mice showed significant activation of microglia (Iba-1) and astrocytes (GFAP) in the CA1 and DG subregions of the hippocampus (Figures 2(a) and 2(b)). In contrast, cotreatment with BAI remarkably inhibited microglial and astrocyte activation, as shown by immunohistochemistry (Figures 2(a) and 2(b)). Similarly, the western blot results showed that LPS significantly increased the expression of Iba- 1 and GFAP in the hippocampus, whereas BAI suppressed this expression (Figures 2(c) and 2(e)). Furthermore, compared with the control mice, LPS-injected mice exhibited a significant increase in IL- $1 \beta$ and TNF- $\alpha$ levels in the hippocampus (Figures 2(a) and 2(e)). Similar to the previous results, the increased levels of IL- $1 \beta$ and TNF- $\alpha$ were largely reduced by BAI coadministration (Figures 2(a) and 2(e)). These results further support the notion that BAI modulates LPS-induced glial activation as well as the levels of the proinflammatory cytokines, IL- $1 \beta$ and TNF- $\alpha$, in the mouse hippocampus.
3.3. BAI Upregulates SIRT1 and Downregulates HMGB1 Expression in Microglia In Vivo and In Vitro. A growing body of evidence supports an important role for SIRT1 in cognitive dysfunction and the inflammatory response [14, 37]. To examine whether the SIRT1 protein level is modulated in the LPS-exposed mouse hippocampus, we immunostained SIRT1 in microglia $\left(\mathrm{Iba}-1^{+}\right)$in hippocampal slices of LPStreated mice. We observed impaired SIRT1 protein expression in microglia exposed to LPS, which was restored by BAI treatment (Figure 3(a)). These results were further confirmed by western blotting (Figure 3(b)). HMGB1 may play a key role in triggering the inflammatory cascade leading to CNS dysfunction. Elevated HMGB1 levels after surgery are associated with compromised blood-brain barrier (BBB) and subsequent neuroinflammation [38]. Immunofluorescence staining of Iba-1 and HMGB1 as well as immunoblotting for HMGB1 detection demonstrated that HMGB1 largely increased in the activated microglia in the hippocampus 1 day after LPS exposure (Figures 3(c) and 3(d)). In contrast, HMGB1 expression was inhibited by BAI supplementation (Figures 3(c) and 3(d)). These in vivo results indicate that BAI upregulated SIRT1 and downregulated HMGB1 expression in hippocampal microglia.

According to the above results, glial cells, particularly microglia, are LPS-responsive cells in the CNS. To further investigate the protective effect of BAI against LPS-induced neuroinflammation, BV2 microglia were used in vitro. To investigate whether SIRT1 is involved in LPS toxicity in BV2 microglia, we compared SIRT1 protein levels in control cells and cells treated with different LPS concentrations (0.1, $0.25,0.5,1$, and $2 \mu \mathrm{g} / \mathrm{ml}$; Figure $4(\mathrm{a}))$. SIRT1 protein level decreased after the LPS challenge (Figure 4(a)). To determine the optimal BAI concentration, we examined the viability of BV2 cells treated with vehicle (1\% DMSO) or various BAI concentrations for $24 \mathrm{~h}$ (Figure $4(\mathrm{~b})$ ). Treatment with up to $40 \mu \mathrm{M}$ BAI did not elicit cytotoxic effects on BV2 cells (Figure 4(b)). Next, we examined the effect of BAI treatment on SIRT1 expression. BAI $(20,40,80$, and $100 \mu \mathrm{M})$ obviously upregulated SIRT1 expression in BV2 cells (Figure 4(c)), in a time-dependent manner (Figure 4(d)). Thus, we selected $40 \mu \mathrm{M}$ BAI as the optimal concentration for subsequent experiments.

BV2 microglia were treated with vehicle (1\% DMSO) or $40 \mu \mathrm{M}$ BAI for $2 \mathrm{~h}$, followed by $100 \mathrm{ng} / \mathrm{ml} \mathrm{LPS}$ or PBS for $24 \mathrm{~h}$. Consistent with the in vivo experiments, SIRT1 expression was markedly low in LPS-exposed BV2 cells (Figure 4(e)), and this effect was reversed by BAI treatment (Figure 4(e)). Additionally, BAI+LPS cotreatment greatly downregulated the level of HMGB1 release compared with the LPS-treated BV2 cells (Figure 4(e)). HMGB1 has been reported to translocate from the nucleus to the cytoplasm in response to inflammatory signals such as LPS. Furthermore, inflammation-induced acetylation of HMGB1 is a vital regulator of its release to the extracellular compartment, where it is a substrate of SIRT1 [18]. Therefore, we examined whether BAI affects this LPS-induced HMGB1 acetylation and translocation in BV2 microglial cells by western blot and immunofluorescence analyses (Figures 4(f)-4(h)). HMGB1 translocation to the cytoplasm increased in cells 


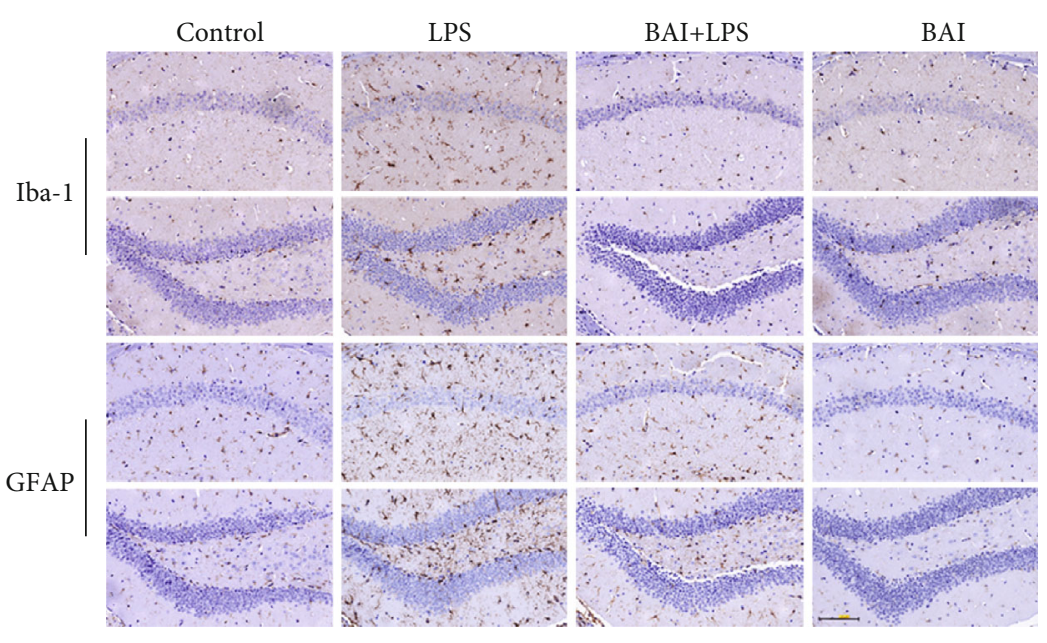

(a)

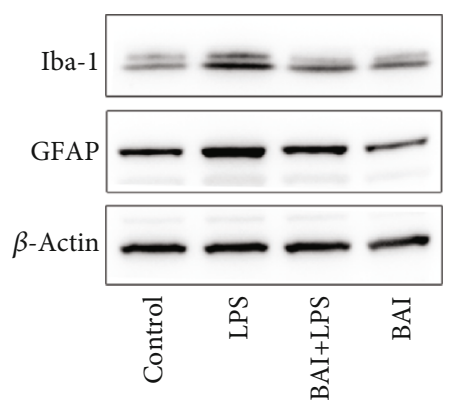

(c)

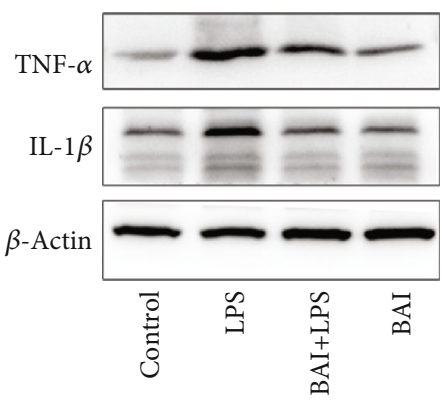

(d)
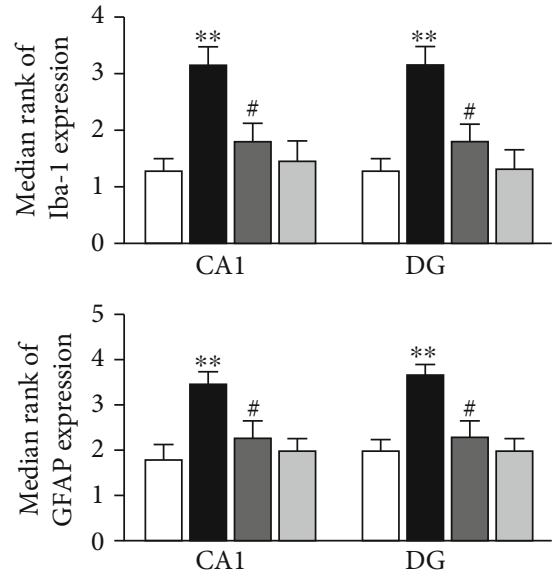

(b)

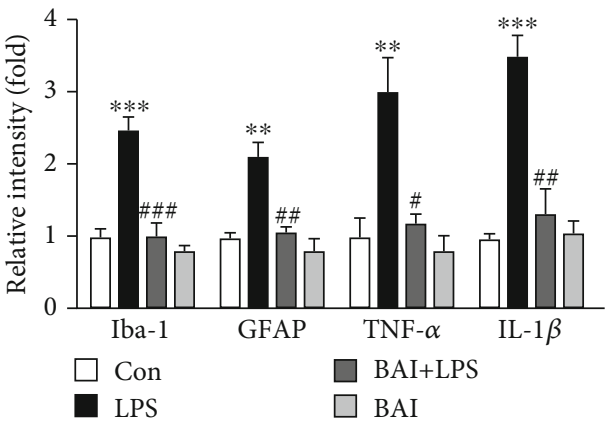

(e)

FIGURE 2: BAI attenuated LPS-induced activated glial cells and the inflammatory response in the mouse hippocampus. (a) Representative photomicrographs of the immunohistochemical analysis of microglia (Iba-1-positive cells) and astrocytes (GFAP-positive cells) in the hippocampal CA1 and DG subregions of mice $24 \mathrm{~h}$ after LPS administration and (b) their statistical analysis (scale bars: $100 \mu \mathrm{M} ; n=6 /$ group). (c) Immunoblotting was used to determine the Iba-1 and GFAP protein levels in hippocampal tissue lysates. (d) IL-1 $\beta$ and TNF- $\alpha$ expression in the hippocampus was determined by immunoblotting $24 \mathrm{~h}$ after LPS and BAI administration. (e) Quantification of the data shown in $\mathrm{c}$ and $\mathrm{d}$. Data are presented as the mean \pm SEM $\left(n=6\right.$ per group). ${ }^{* *} P<0.01,{ }^{* * *} P<0.001$, control vs. LPS; ${ }^{\#} P<0.05,{ }^{\#} P<0.01$,

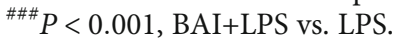

exposed to LPS; however, these changes were restored by BAI treatment (Figures $4(\mathrm{~g})$ and $4(\mathrm{~h})$ ). Furthermore, when BV2 cells were stimulated with LPS for $4 \mathrm{~h}$, the level of acetylated HMGB1 was significantly enhanced; however, BAI cotreatment with LPS markedly reversed this effect (Figure 4(f)). Our data confirmed that BAI treatment induced SIRT1 expression and suppressed HMGB1 acetylation and translocation, thereby counteracting the effects of LPS in mice and BV2 cells.

\subsection{BAI Neuroprotection against LPS-Induced Cognitive} Deficits Is SIRT1 Dependent. To explore whether the neuroprotective effect of BAI is SIRT1 dependent, we pretreated mice with EX-527, a specific SIRT1 inhibitor. The LPS group and LPS+EX-527 group exhibited longer escape latencies, fewer platform crossings, and shorter target quadrant times than the control mice on days 3 and 4 (Figures $5(\mathrm{a})-5(\mathrm{c})$ )). There was no difference between the LPS group and the LPS+EX-527 group (Figures 5(a)-5(c)). In contrast, in the BAI+LPS group, the latencies to find the platform on day 4 of training were shorter and the frequency of crossing the platform and the time spent in the target quadrant significantly increased (Figures 5(a)-5(c)). Interestingly, EX-527 treatment affected the BAI inhibitory effect on cognition decline. Administration of EX-527 prior to LPS and BAI treatment abolished the protective effects of BAI (Figures 5(a)-5(c)). Pretreating BAI+LPS mice with Ex-527 extended their latency to find the platform on day 4 of training (Figure 5(a)). In the probe test, the platform-crossing times and the time spent in the target quadrant were significantly shorter in the BAI+LPS+EX-527 group than in the BAI+LPS group (Figures 5(b) and 5(c)). These results indicate that BAI pretreatment was effective in improving spatial learning and memory of LPS-treated mice in a SIRT1dependent manner.

We then examined the effect of BAI-mediated SIRT1 activation on inflammation in vivo using LPS-induced mice. The results showed that the BAI-inhibited glial cell activation was abolished by EX-527 treatment. Compared with the LPS and LPS+EX5-27 groups, BAI administration decreased the 


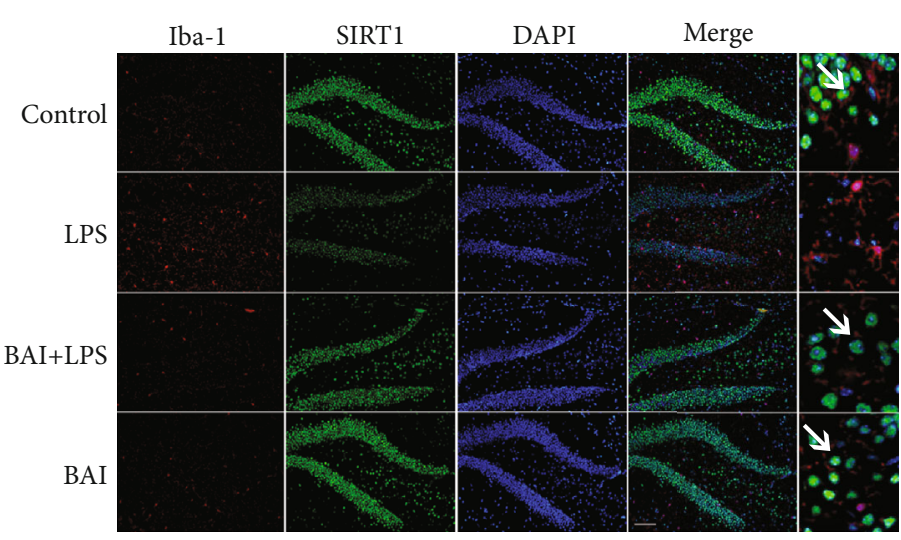

(a)

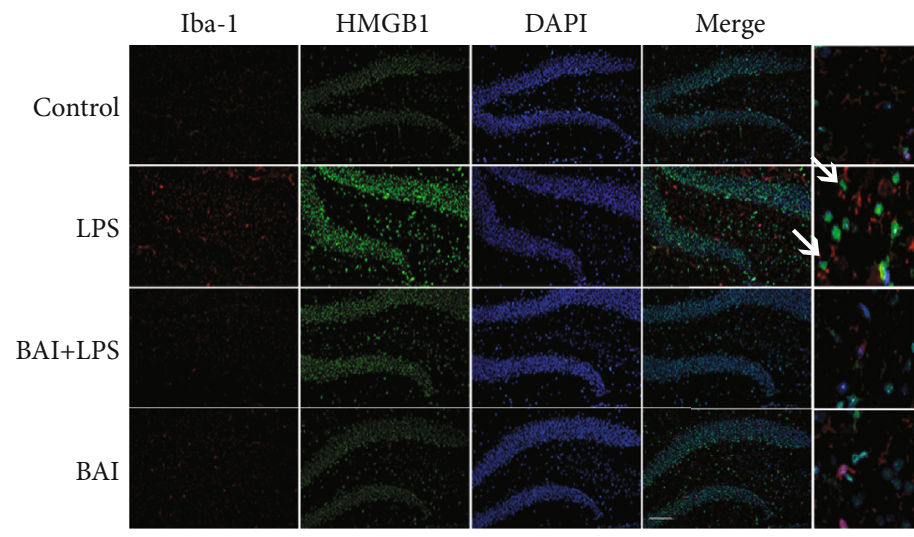

(c)

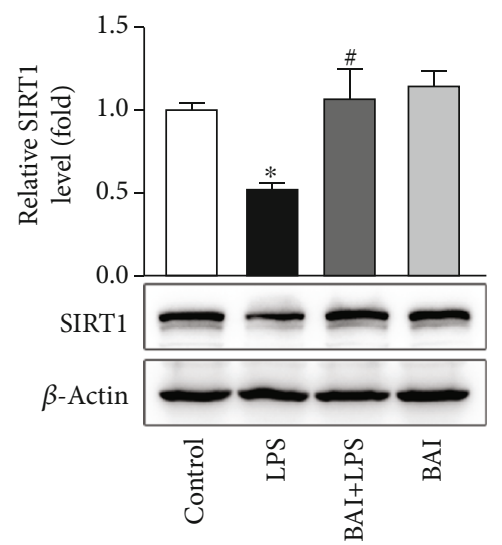

(b)

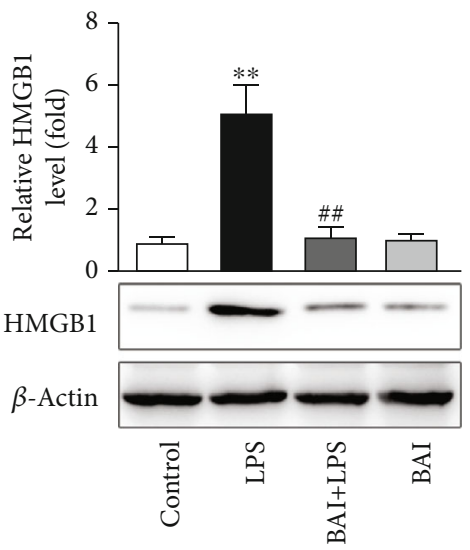

(d)

FIgURE 3: BAI upregulated SIRT1 expression and downregulated HMGB1 expression in mice with hippocampal impairment. (a) Immunofluorescence of SIRT1 (green) in microglial cells (Iba-1+; red) in the mouse hippocampus $24 \mathrm{~h}$ after LPS exposure. Arrows point to SIRT1-positive microglial cells (Iba-1+). (b) Western blot analysis of SIRT1 expression in mice subjected to LPS and BAI. (c) Immunofluorescence of HMGB1 (green) in microglial cells (Iba-1+; red) in the hippocampus $24 \mathrm{~h}$ after LPS exposure. Arrows point to HMGB1-positive microglial cells (Iba-1+). (d) Western blot analysis of HMGB1 expression in mice subjected to LPS and BAI. Data are expressed as the mean \pm SEM $(n=4-5)$. ${ }^{*} P<0.05,{ }^{* *} P<0.01$, control vs. LPS; ${ }^{\#} P<0.05,{ }^{\#} P<0.01$, BAI+LPS vs. LPS. SIRT1, silent information regulator 1; HMGB1, high-mobility group box protein 1 .

expression of Iba-1 and GFAP in the hippocampus, whereas pretreatment of the BAI+LPS-treated mice with EX-527 increased their expression (Figures 5(d) and 5(f)). Furthermore, we observed that the expression levels of TNF- $\alpha$ and IL- $1 \beta$ in the hippocampus were increased in the LPS and LPS+EX-527 groups relative to the control group, and there was no difference in the expression of these proteins between the LPS and LPS+EX-527 groups (Figures 5(e) and 5(f)). In contrast, BAI significantly suppressed TNF- $\alpha$ and IL- $1 \beta$ expression relative to mice subjected to LPS, which was reversed by topical EX-527 treatment (Figures 5(e) and 5(f)). Taken together, our results suggest that BAI effectively ameliorated the memory deficits and inhibited microglial activation and proinflammatory cytokines' expression induced by systemic LPS treatment through a SIRT1-dependent mechanism in the hippocampus.
3.5. HMGB1, A SIRT1 Downstream Molecule, Participates in the Microglial Protective Action of BAI against LPS-Induced Neuroinflammation. HMGB1 as a downstream mediator of systemic inflammation has opened a new avenue for the development of anti-inflammatory therapeutics. SIRT1 dissociates from HMGB1 during translocation from the nucleus to the cytosol and promotes the HMGB1 acetylation $[39,40]$. SIRT1-dependent HMGB1 deacetylation regulates microglial polarization and the subsequent neuroinflammatory response [41]. To analyze whether the BAI-mediated deactivation of the HMGB1 signaling pathway is SIRT1 dependent, we used EX-527, in vivo (Figures 6(a) and 6(b)). The immunoblot results indicated that BAI treatment reduced LPSinduced HMGB1 expression, whereas SIRT1 inhibition in the BAI+LPS group resulted in upregulated HMGB1 expression (Figure 6(b)). Similarly, we conducted a double 


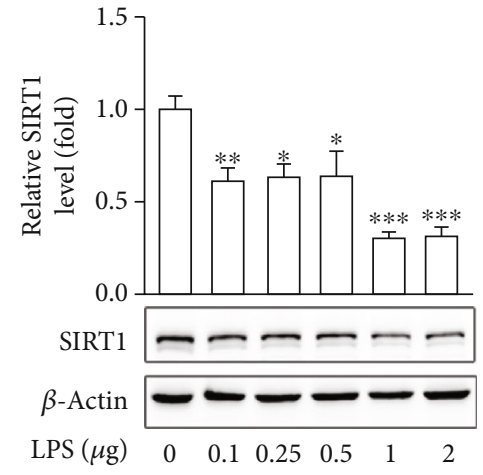

(a)

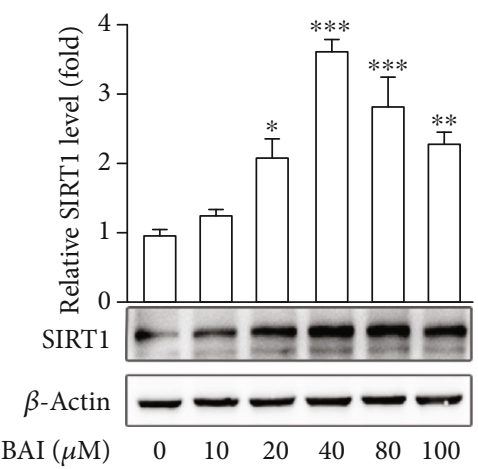

(c)

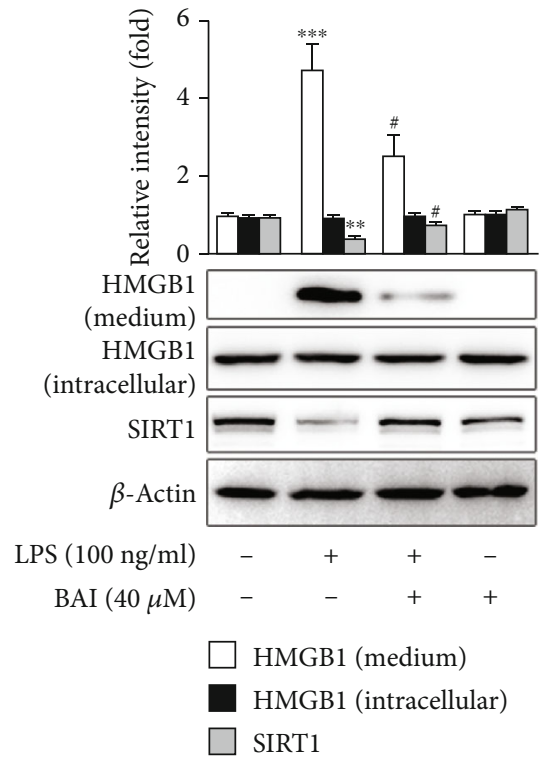

(e)

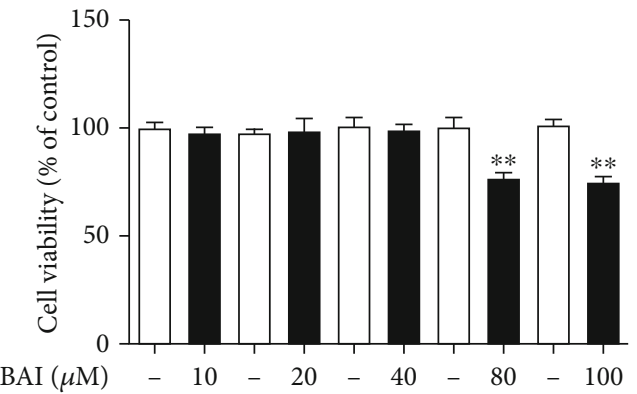

(b)

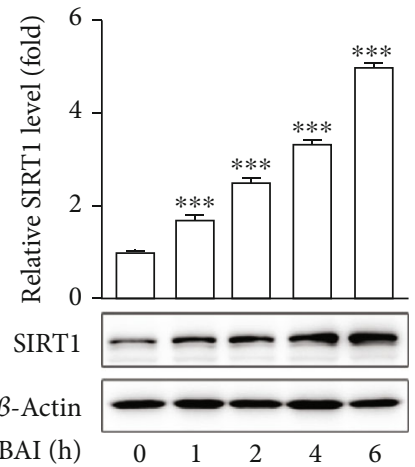

(d)

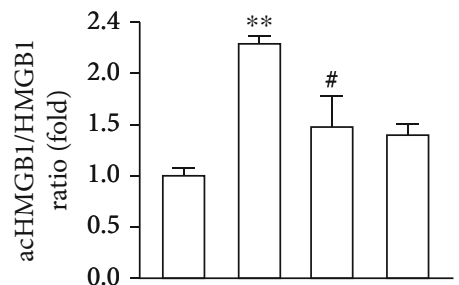

IP:HMGB1

IB:acetylated lysine

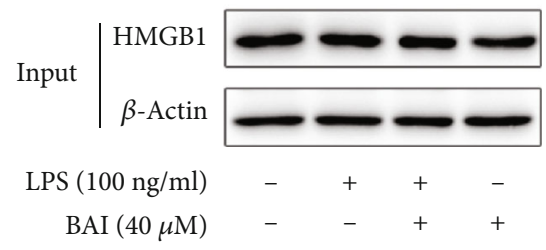

(f)

Figure 4: Continued. 


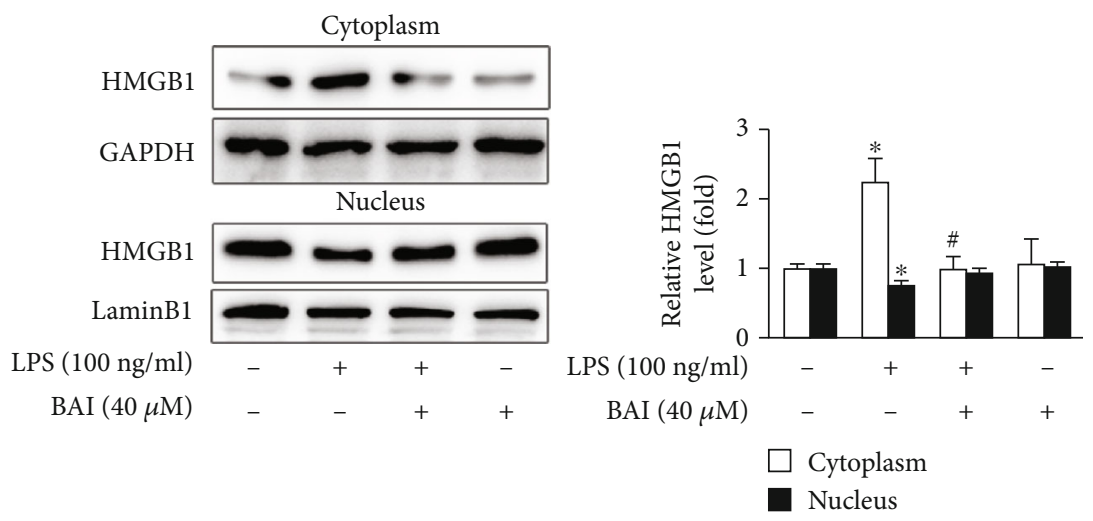

(g)

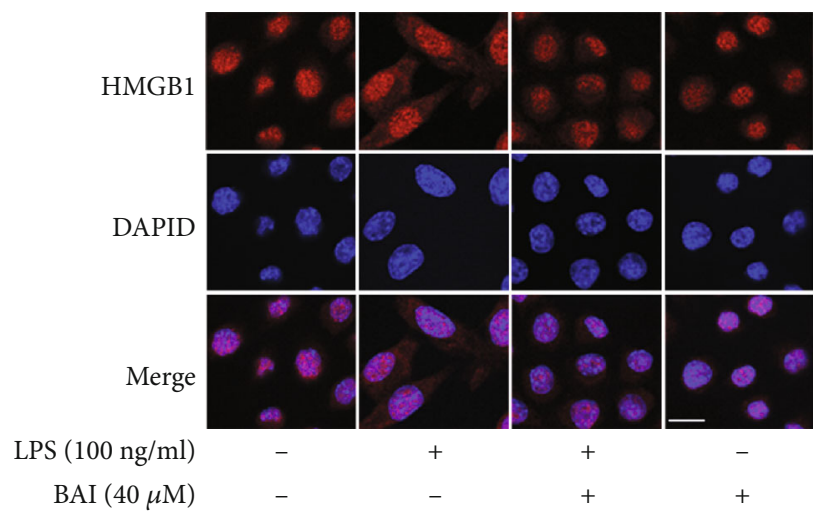

(h)

FIGURE 4: BAI significantly increased SIRT1 expression and decreased HMGB1 release in LPS-induced BV2 microglia. (a) BV2 cells were stimulated with various concentrations of LPS $(0.1,0.25,0.5,1$, and $2 \mu \mathrm{g} / \mathrm{ml})$ for $24 \mathrm{~h}$, followed by western blot analysis of SIRT1 protein. (b) BV2 cells were cultured with the indicated BAI concentrations $(10,20,40,80$, and $100 \mu \mathrm{M})$ for $24 \mathrm{~h}$, and cell viability was determined by CCK8. (c, d) BV2 cells were cultured with different BAI concentrations for $24 \mathrm{~h}$ (c) or with $40 \mu \mathrm{M}$ BAI for different durations (1, 2, 4, and $6 \mathrm{~h}$ ) (d), and SIRT1 levels were analyzed by immunoblotting. (e) BV2 cells were pretreated with $40 \mu \mathrm{M}$ BAI for $2 \mathrm{~h}$, followed by $100 \mathrm{ng} / \mathrm{ml}$ LPS stimulation for $24 \mathrm{~h}$. Western blot analysis of the levels of SIRT1, secreted HMGB1, and intracellular HMGB1 was performed. (f) BV2 cells were preincubated with $40 \mu \mathrm{M}$ BAI for $2 \mathrm{~h}$ and then stimulated with $100 \mathrm{ng} / \mathrm{ml} \mathrm{LPS} \mathrm{for} 4 \mathrm{~h}$. Acetylated lysine and HMGB1 expression levels were determined by immunoprecipitation. (g) BV2 cells were pretreated with $40 \mu \mathrm{M}$ BAI for $2 \mathrm{~h}$, followed by $100 \mathrm{ng} / \mathrm{ml}$ LPS treatment for $6 \mathrm{~h}$. The nucleocytoplasmic translocation of HMGB1 was determined by western blot analysis. GAPDH and LaminB1 were used as loading controls for the cytoplasmic and nuclear fractions, respectively. (h) Immunofluorescence of the HMGB1 (red) cytosolic translocation was conducted with confocal scanning microscopy. Scale bar $=10 \mu \mathrm{M}$. All data were normalized to the control group and are presented as means $\pm \operatorname{SEM}(n=3) .{ }^{*} P<0.05,{ }^{* *} P<0.01,{ }^{* *} P<0.001$, control vs. LPS; ${ }^{*} P<0.05$, BAI+LPS vs. LPS.

immunofluorescent staining to assess HMGB1 expression in microglia $\left(\mathrm{Iba}-1^{+}\right)$in the hippocampus after LPS exposure (Figure 6(a)). The results showed that the LPS-induced increase in HMGB1 protein expression in microglia was SIRT1 dependent and that BAI significantly decreased the expression of HMGB1 and Iba-1 proteins in hippocampal microglia from LPS-treated mice (Figure 6(a)). This finding suggests that BAI increased the level of SIRT1 and inhibited the activation of the HMGB1 signaling pathway, which alleviated the activation of microglia and mitigated neuroinflammation.

To further clarify that the BAI-modulated downregulation of HMGB1 release was attributed to the increased expression of SIRT1, we manipulated the expression and activity of SIRT1 using siRNA. SIRT1 was knocked down in BV2 cells by siRNA-1 or siRNA-2 transfection
(Figure $7(\mathrm{a})$ ). Similar to the in vivo results, the protective effect of BAI on microglia against LPS exposure was abolished in siRNA-1 or siRNA-2-transfected BV2 cells, indicating that BAI could no longer reverse the upregulation of HMGB1 release in LPS-treated cells (Figures 7(b) and 7(c)). We further analyzed the subcellular localization of HMGB1 and the expression of acetylated HMGB1 (Figures $7(\mathrm{~d})-$ 7(h)). The immunoblot analysis indicated that SIRT1 knockdown in cultured siRNA-1 or siRNA-2-transfected BV2 cells incubated with BAI and LPS resulted in higher acetylation of HMGB1 (Figures 7(d) and 7(e)) and enhanced nucleus-tocytoplasm translocation (Figures $7(\mathrm{~g})$ and $7(\mathrm{~h})$ ). Moreover, the subcellular localization of HMGB1 was confirmed by immunofluorescence staining (Figure 7(f)), which showed that BAI-inhibited HMGB1 release under LPS stimulation was abrogated by SIRT1 silencing. 


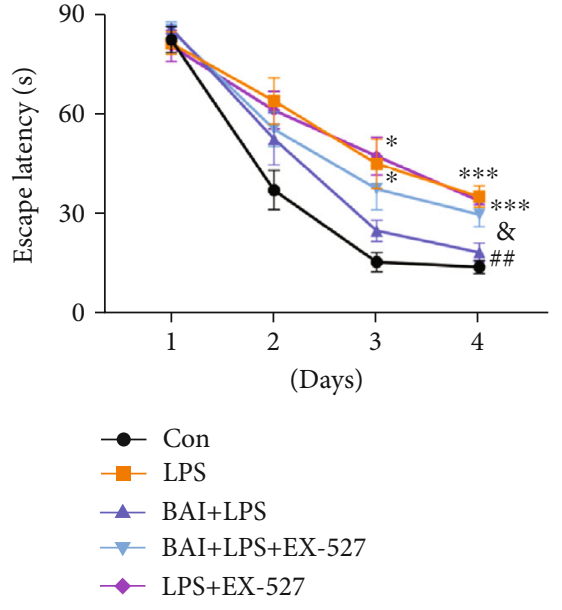

(a)

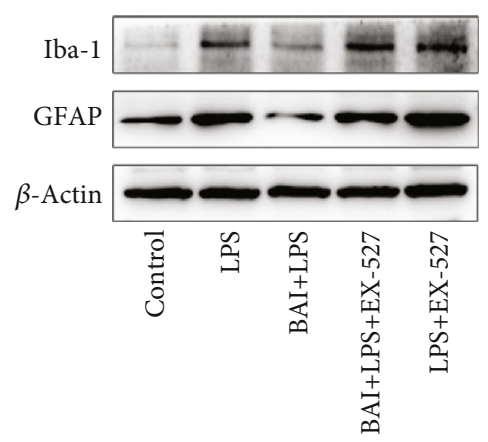

(d)
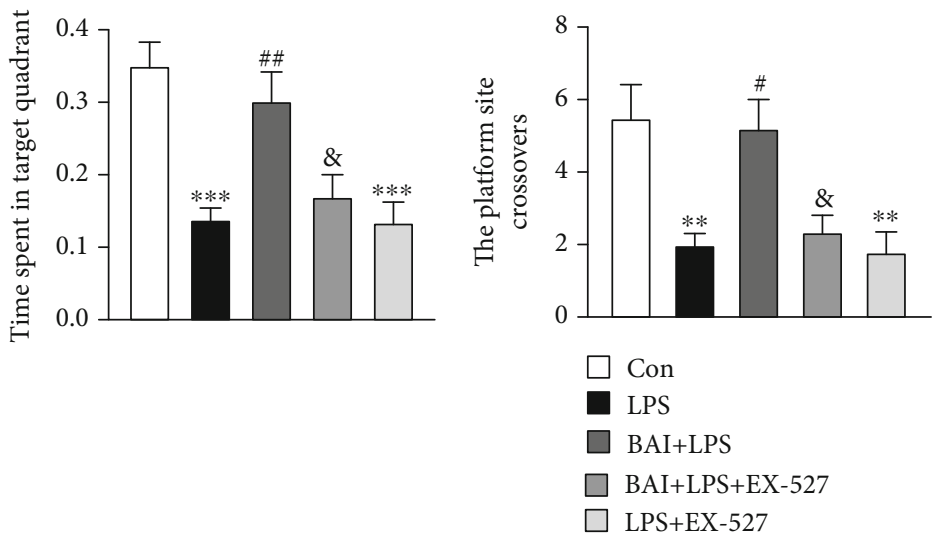

(b)

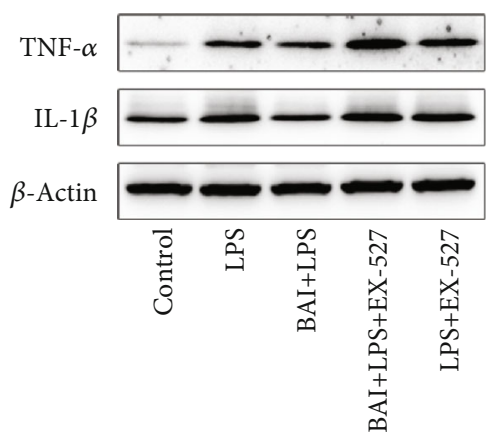

(e) (c)

FIGURE 5: BAI-improved neuroinflammation and neurocognitive impairment induced by exposure to LPS in mice are SIRT1 dependent. Mice were divided into five groups: control, LPS, BAI+LPS, BAI+LPS+EX-527, and LPS+EX-527. (a) Latency to the platform during spatial working memory testing. (b) Time spent in the target quadrant during probe testing. (c) Platform-site crossings during the probe trial. Data are expressed as the mean \pm SEM $(n=8-10)$. (d) Western blot analysis of Iba-1 and GFAP in the hippocampus following LPS and BAI treatment, with or without EX-527 treatment. (e) Immunoblots of the inflammatory cytokines, IL-1 $\beta$ and TNF- $\alpha$. (f) Quantification of the data shown in $d$ and e. Data are expressed as the mean \pm SEM $\left(n=5\right.$ /group) ${ }^{*} P<0.05,{ }^{* *} P<0.01$, ${ }^{* * *} P<0.001$, control vs. LPS or LPS+EX-527; ${ }^{\#} P<0.05,{ }^{\# \#} P<0.01,{ }^{\# \#} P<0.001$, BAI+LPS vs. LPS; ${ }^{\circledR} P<0.05,{ }^{\& \&} P<0.01$, BAI+LPS vs. BAI+LPS+EX-527. EX-527, a specific SIRT1 inhibitor.

\section{Discussion}

The current study was conducted to examine the effects of BAI on cognitive dysfunction induced by a single systemic dose of LPS injection in mice and assess the changes in neuroinflammation and behavior. We found that BAI improved cognitive dysfunction induced by intraperitoneal administration of LPS. The protective effects of BAI on cognitive impairment in mice appeared to be related to its antiinflammatory activity in microglial cells, modulating the activity of SIRT1, as well as downregulating the HMGB1 signaling pathway (Figure 8).

Several studies have shown that LPS-induced systemic inflammation impaired learning and memory $[7,8,32]$ through increasing glial cell activation, production of proinflammatory mediators, and $\mathrm{A} \beta$ accumulation. Notably, LPS-induced inflammation and pattern recognition recep- tors expressed at the BBB surface can lead to endothelial inflammation and subsequent neuroinflammation [6]. Single systemic LPS-induced cytokines and alarmins in the serum also directly disrupt the BBB and alter BBB function, including adsorptive transcytosis, immune cell trafficking, and various transport functions, thus allowing proinflammatory immune cells and molecules to the CNS [42, 43]. Neuroinflammation is characterized by the activation of CNS resident microglia and expression of proinflammatory mediators, and is a pivotal feature in virtually every neurological cognitive impairment [6]. Indeed, reactive microglia can induce cytokine release and astrocyte activation [44], which leads to inflammatory toxicity and neuronal cell death [6]. Furthermore, the activity of astrocytes, another important type of glial cells in the brain, may exacerbate inflammatory reactions and tissue damage. Age, trauma, infection, and autoimmune response increase the number of astrocytes and induce 


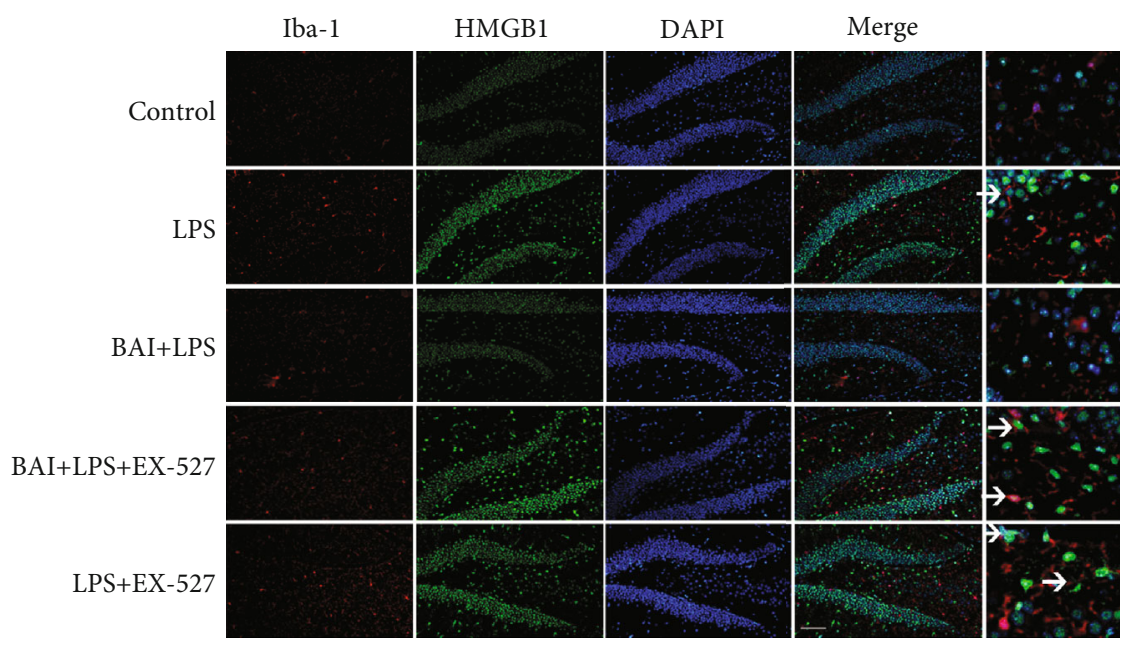

(a)
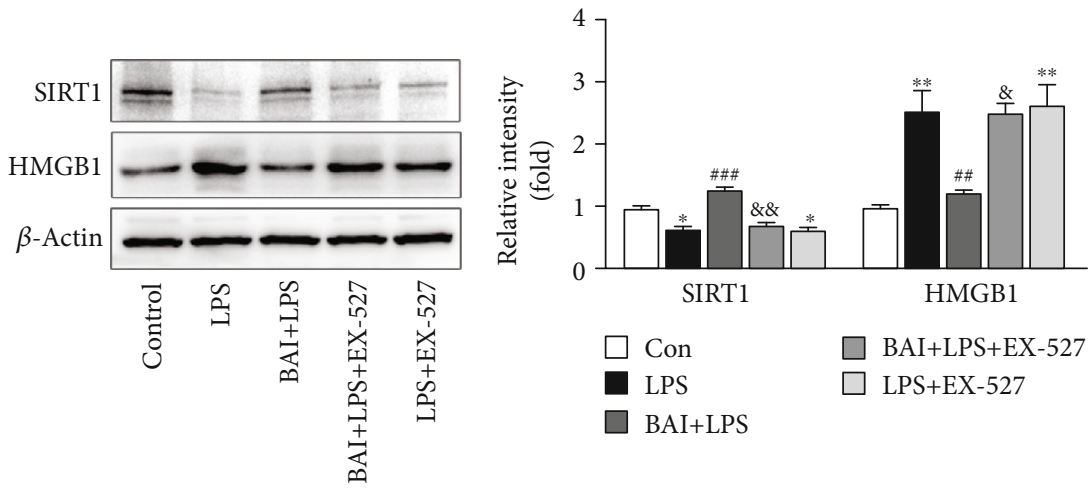

(b)

FIGURE 6: BAI suppressed the HMGB1 pathway by elevating SIRT1 in the hippocampus of LPS-induced mice. (a) HMGB1 immunofluorescence (green) in microglial cells (Iba-1+; red) counterstained with DAPI (blue), following LPS and BAI treatment; scale bars $=100 \mu \mathrm{M}$. Arrows point to HMGB1-positive microglial cells (Iba-1+). (b) Western blot analysis confirming the effect of BAI and EX527 on SIRT1 and HMGB1 protein expression in LPS-induced mice. Data are expressed as the mean \pm SEM $(n=5 /$ group $) .{ }^{*} P<0.05,{ }^{* *} P$ $<0.01$, control vs. LPS or LPS+EX-527; ${ }^{\# \#} P<0.01,{ }^{\# \# \#} P<0.001$, BAI+LPS vs. LPS; ${ }^{\circledR} P<0.05,{ }^{\& \&} P<0.01$, BAI+LPS vs. BAI+LPS+EX-527.

rapid synthesis of GFAP [45]. Our results demonstrated that BAI mitigates LPS-induced increase of Iba- 1 and GFAP and participates in the modulation of microglia and astrocyte activations, which is consistent with previous studies [21, 29]. Activated microglia are the main cellular source of central proinflammatory cytokines; hence, we focused on microglia in this study. By the same token, we used systemic LPS injection into rodents as it has been extensively used as a model for studying the interaction between inflammation and cognitive deficits.

BAI is an FDA-approved medical food flavonoid. Numerous studies have demonstrated that some natural phytochemicals have neuroprotective effects including on learning and memory impairment [46]. BAI has been assessed for safety, toxicity, and tolerability properties in in vivo and in vitro studies [47]. Thus far, a wide array of neuroprotective effects of BAI on neurodegenerative diseases such as ischemic stroke, Alzheimer's disease, Parkinson's disease, and traumatic brain injury have been reported by different studies. In addition, BAI exhibits considerable anxiolytic-like and antidepressant effects. It has been revealed that BAI has sev- eral pharmacological activities, including antioxidant, antiinflammatory, antiapoptotic, and antiexcitotoxicity properties [21]. BAI and its metabolites have been shown to induce SIRT1 expression and activation [12]. Herein, we examined the potential of BAI as an agent for LPS impairment therapy. In terms of results obtained in this study, both LPS-induced injury and the protective effect of BAI may play a dual role in the periphery and CNS. To elucidate the roles of SIRT1 in the BAI protective effects on LPS-impaired microglial action and cognition, we used SIRT1 inhibitor EX-527 in LPS-induced mice. In the absence of SIRT1, the BAI effects on cognition dysfunction and neuroinflammation were largely attenuated. These results were further confirmed in the in vitro experiments with SIRT1 siRNA in microglial BV2 cells.

SIRT1 is an NAD-dependent deacetylase mainly involved in stress responses, cellular metabolism, and aging [9]. Its levels in the brain, especially in the hippocampus, are obviously higher than those in other tissues in mammals [11]. Compelling evidence has indicated that SIRT1 plays an important role in neurodegenerative diseases and cognitive 


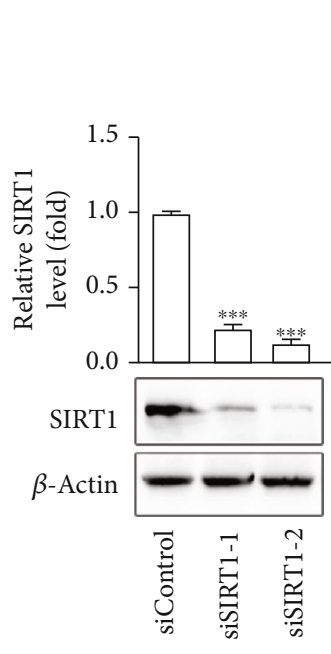

(a)

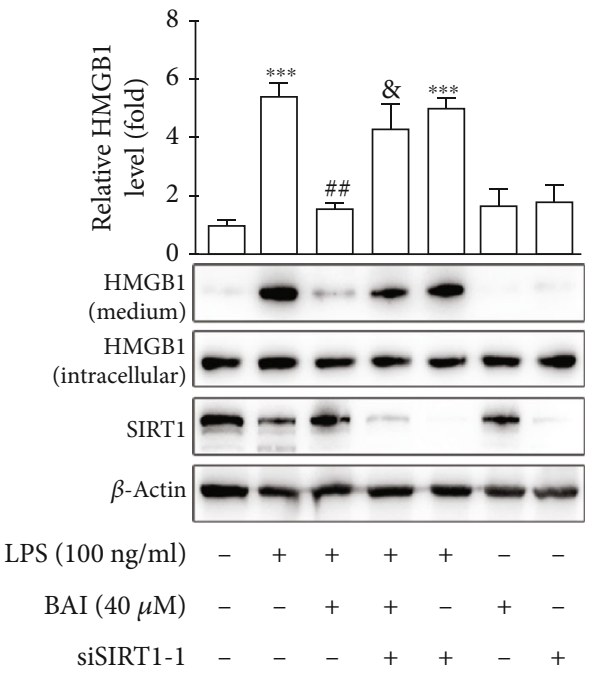

(b)

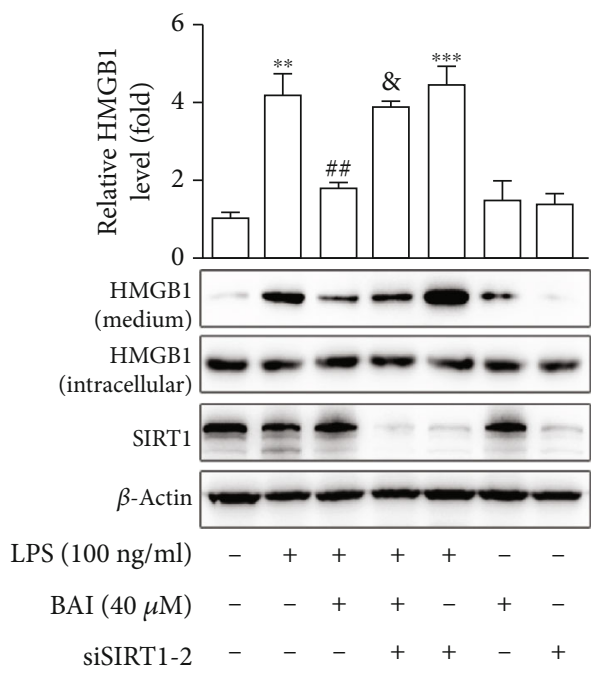

(c)

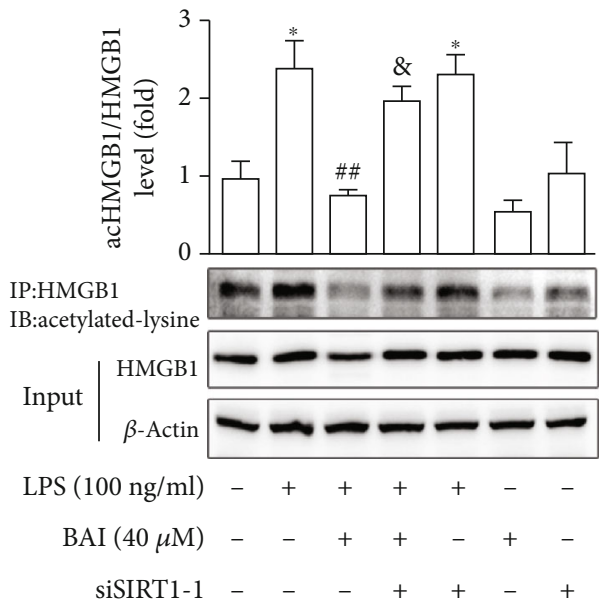

(d)

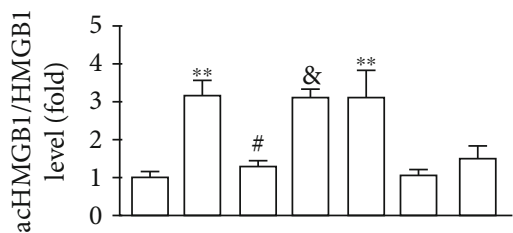

IP:HMGB1

IB:acetylated lysine

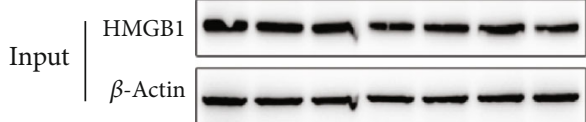

LPS (100 ng/ml)

BAI $(40 \mu \mathrm{M})$ siSIRT1-1

(e)

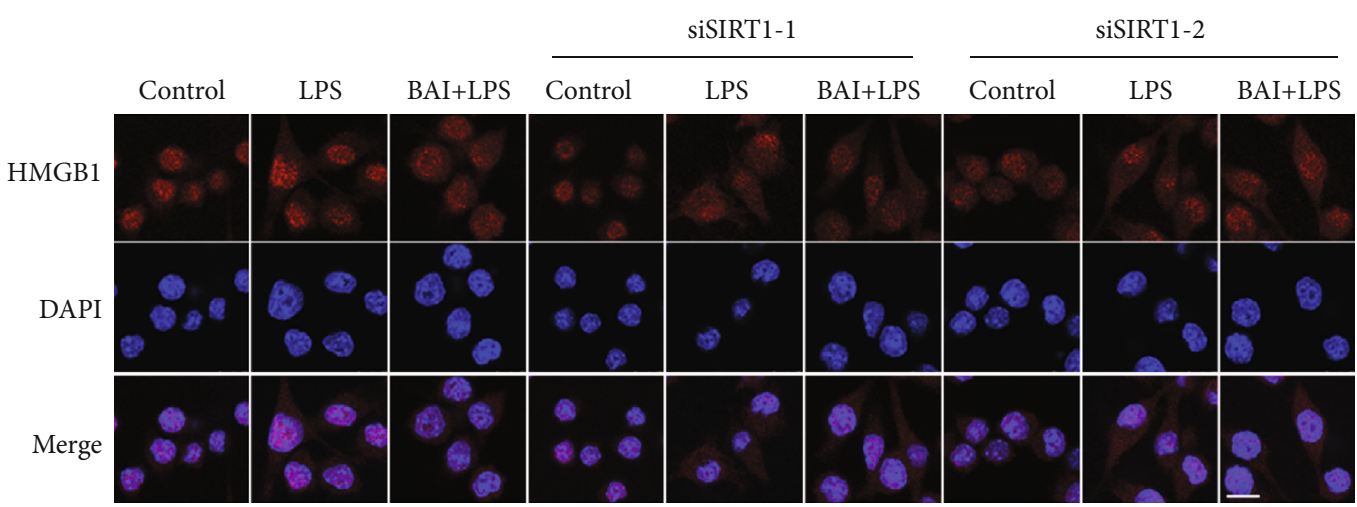

(f)

FIgURE 7: Continued. 


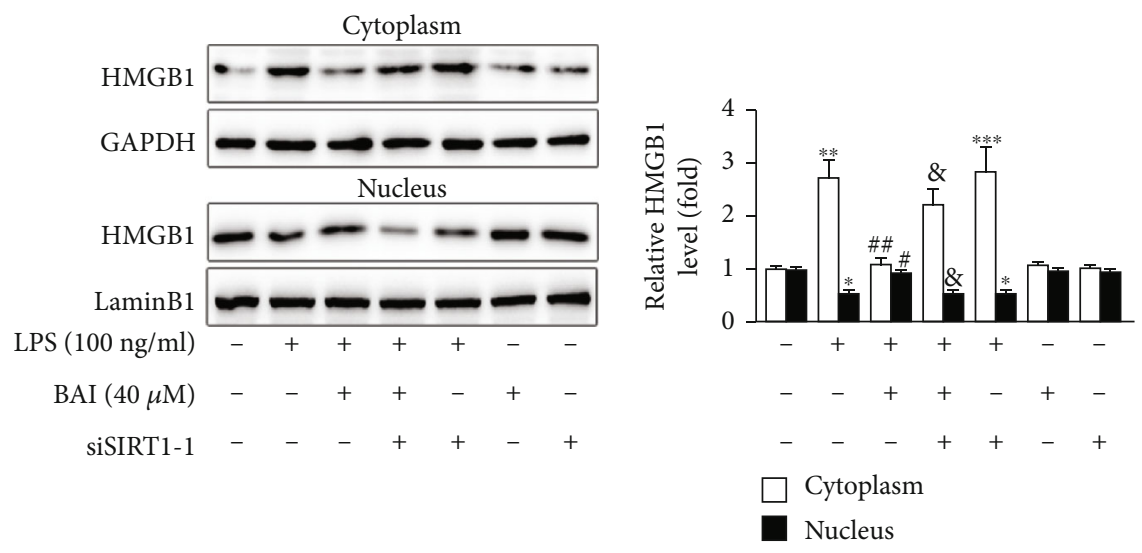

(g)
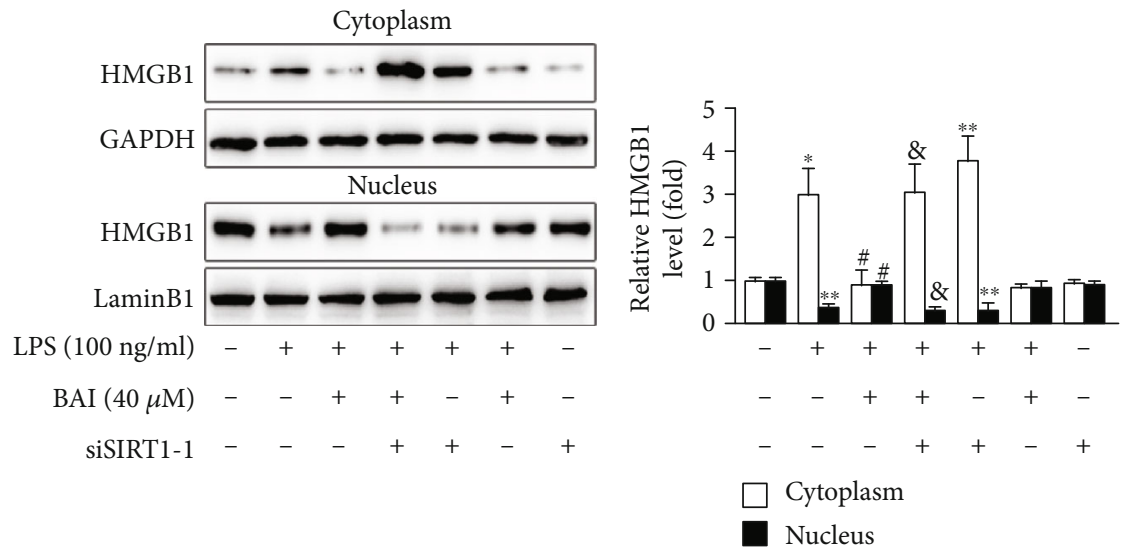

(h)

FIgURE 7: SIRT1 is required for BAI inhibition of LPS-induced HMGB1 release from BV2 microglial cells. BV2 cells were transfected with SIRT1-targeted siRNA-1, siRNA-2, or control siRNA. (a) SIRT1 protein levels in siRNA-1 or siRNA-2-transfected BV2 cells were measured by immunoblotting. (b, c) After transfection for $36 \mathrm{~h}$, cells were exposed to LPS in the presence or absence of BAI for $24 \mathrm{~h}$, and the expression of SIRT1, secreted HMGB1, and intracellular HMGB1 was measured by western blotting. (d, e) After transfection, BV2 cells were cultured in LPS medium alone or with $40 \mu \mathrm{M}$ BAI for $4 \mathrm{~h}$. Coimmunoprecipitation analysis showed the level of acetylated HMGB1. (f) Cells transfected with SIRT1-targeted siRNA-1 or siRNA-2 for $36 \mathrm{~h}$ were exposed to LPS in the presence or absence of BAI for $6 \mathrm{~h}$; immunofluorescent images of the HMGB1 (red) cytoplasmic translocation were observed with confocal scanning microscopy. Scale bar: $10 \mu \mathrm{M}$. (g, h) HMGB1 levels in the cytoplasmic and nuclear fractions were analyzed by western blotting. GAPDH and LaminB1 were used as loading controls for cytoplasmic and nuclear fractions, respectively. All the results are presented as means \pm SEM of three independent experiments. ${ }^{*} P<0.05,{ }^{* *} P<0.01,{ }^{* * *} P<0.001$, control vs. LPS or LPS + siRNA- $1 / 2 ;{ }^{\#} P<0.05$, ${ }^{\# \#} P<0.01$, BAI+LPS vs. LPS; ${ }^{\&} P<0.05, \mathrm{BAI}+\mathrm{LPS}$ vs. BAI+LPS+siRNA-1/2.

dysfunction [48-50]. Furthermore, SIRT1 inhibits NF- $\kappa$ B signaling activation and the resultant production of proinflammatory cytokines and protects neuronal PC12 cells from caspase-3-dependent apoptosis [49]. SIRT1 activation by resveratrol has been found to inhibit matrix metalloproteinases, regulate BDNF signaling, and reduce the production of a wide range of cytokines [48, 51]. Consistently, we demonstrated here that the level of SIRT1 protein was dramatically decreased in microglial cells in the hippocampus after LPS exposure, and this effect was reversed by BAI. In addition, SIRT1 is one of the downstream pathways of adenosine monophosphate-activated protein kinase (AMPK) activation. AMPK increases the intracellular $\mathrm{NAD}^{+} / \mathrm{NADH}$ ratio to activate the $\mathrm{NAD}^{+}$-dependent type III deacetylase SIRT1 [52]. BAI attenuates hyperglycemia aggravated ischemia/reperfusion injury in a manner dependent on AMPK [53].
Therefore, we think that BAI may also upregulate SIRT1 in an AMPK-dependent manner in the current study.

The interaction between SIRT1 and HMGB1 has previously been documented during an inflammation response. HMGB1 is a novel deacetylation target of SIRT1 [54], followed by acetylation, nucleus-to-cytoplasm translocation, and release from stressed cells, unleashing a signaling cascade of events leading to neuroinflammation. It is widely accepted that HMGB1 is a key inflammatory mediator in the pathogenesis of cognition dysfunction. Microglial activation and the subsequent inflammatory response in the hippocampus can disrupt neuronal networks required for learning and memory. Reportedly, acetylation of HMGB1 is a key process prior to its nucleus-to-cytoplasm translocation and extracellular secretion from kidney cells, accelerating the development of sepsis-associated acute kidney injury (SA-AKI) 


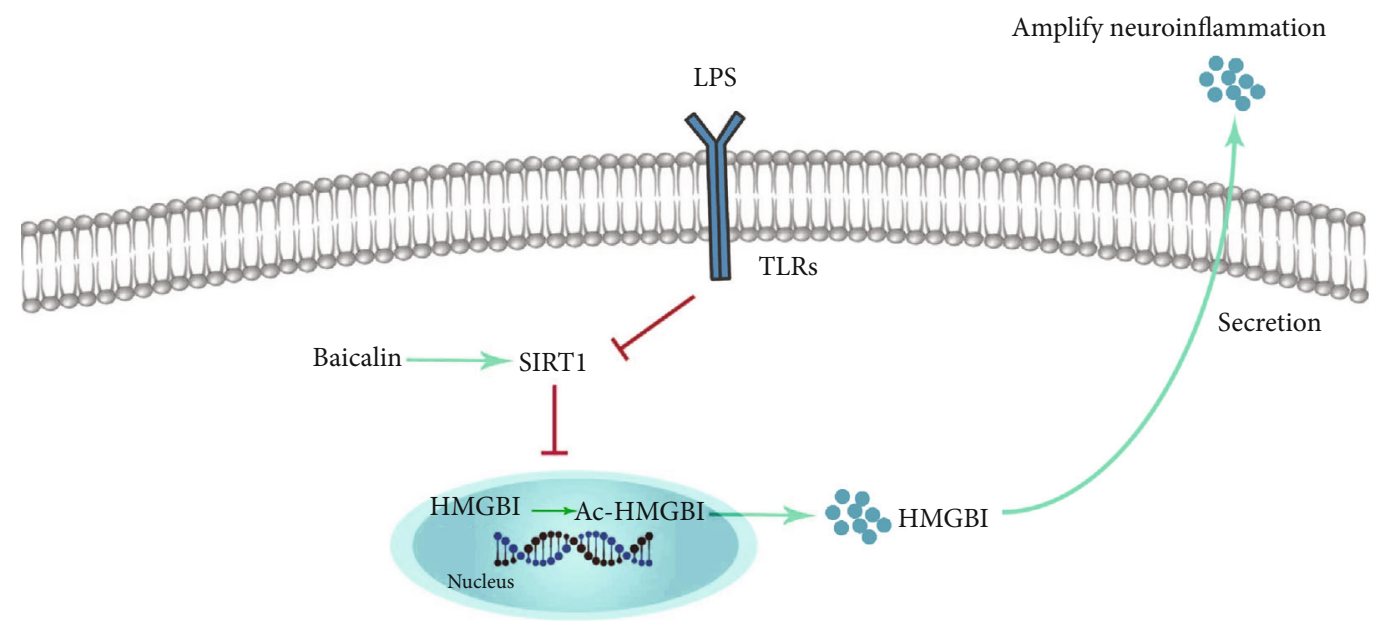

FIGURE 8: Schematic illustration of the possible protective mechanism of BAI administration in LPS-induced HMGB1 release in microglia cells.

[55]. In the present study, we found that LPS-mediated SIRT1 inhibition increased cytosolic HMGB1 levels, while BAI-mediated SIRT1 activation facilitated HMGB1 translocation from the cytoplasm to the nucleus in BV2 cells. Thus, the exact roles of the SIRT1/HMGB1 pathway and its regulatory roles in microglial dysfunction and $\mathrm{dNCR}$ are of great importance.

There are several limitations in the current study. First, we did not measure both intracranial and peripheral cytokine levels at the same time in this study. Second, we only explored the role of SIRT1/HMGB1 signaling in microglial cells. In future studies, the role of this signaling pathway in other cell types such as neurons and astrocytes should be investigated. Finally, our study focused on relatively shortterm cognitive functions (4-5 days after LPS exposure), and the long-term effects of BAI pretreatment remained unexplored.

In summary, we uncovered the SIRT1/HMGB1 pathway by which BAI prevents LPS-induced cognitive dysfunction and neuroinflammation. Defining the detailed mechanisms by which activation of microglial SIRT1 and degradation of HMGB1 protein-mediated the cognitive-improving effect of BAI may indicate a promising therapeutic target for the treatment of dNCR.

\section{Conclusions}

Our study demonstrated that BAI can improve acute neurocognitive impairment and inhibited neuroinflammation in LPS-induced mice via a SIRT1-dependent pathway and involved the inhibition of HMGB1. These data confirmed that BAI may have efficient medicinal value and proposed it as a new potential candidate for dNCR therapy (Figure 8).

\section{Data Availability}

The data used to support the findings of this study are included within the article.

\section{Conflicts of Interest}

The authors declare that there is no conflict of interests regarding the publication of this paper.

\section{Acknowledgments}

This work is funded by the National Natural Science Foundation of China (No. 81873726, 81971012, 81901095, and 81801070), Peking University Clinical Medicine plus X Youth Project (PKU2020LCXQ016), and Key Clinical Projects of Peking University Third Hospital (BYSYZD2019027 and BYSYZD2019029).

\section{References}

[1] L. Evered, B. Silbert, D. S. Knopman et al., "Recommendations for the nomenclature of cognitive change associated with anaesthesia and surgery-2018," Anesthesiology, vol. 129, no. 5, pp. 872-879, 2018.

[2] J. Steinmetz, K. B. Christensen, T. Lund, N. Lohse, and L. S. Rasmussen, "Long-term consequences of postoperative cognitive dysfunction," Anesthesiology, vol. 110, no. 3, pp. 548-555, 2009.

[3] I. B. Hovens, R. G. Schoemaker, E. A. van der Zee, A. R. Absalom, E. Heineman, and B. L. van Leeuwen, "Postoperative cognitive dysfunction: involvement of neuroinflammation and neuronal functioning," Brain, Behavior, and Immunity, vol. 38, pp. 202-210, 2014.

[4] Y. Cao, Z. Li, L. Ma et al., "Isoflurane-induced postoperative cognitive dysfunction is mediated by hypoxia-inducible factor- $1 \alpha$-dependent neuroinflammation in aged rats," Molecular Medicine Reports, vol. 17, no. 6, pp. 7730-7736, 2018.

[5] S. A. Villeda, J. Luo, K. I. Mosher et al., "The ageing systemic milieu negatively regulates neurogenesis and cognitive function," Nature, vol. 477, no. 7362, pp. 90-94, 2011.

[6] S. Subramaniyan and N. Terrando, "Neuroinflammation and perioperative neurocognitive disorders," Anesthesia and Analgesia, vol. 128, no. 4, pp. 781-788, 2019.

[7] M. Satomoto, Z. Sun, Y. U. Adachi, H. Kinoshita, and K. Makita, "Sevoflurane preconditioning ameliorates lipopolysaccharide-induced cognitive impairment in mice," Experimental Animals, vol. 67, no. 2, pp. 193-200, 2018. 
[8] J.-H. Song, J. W. Lee, B. Shim et al., "Glycyrrhizin alleviates neuroinflammation and memory deficit induced by systemic lipopolysaccharide treatment in mice," Molecules, vol. 18, no. 12, pp. 15788-15803, 2013.

[9] W. Zhao, J. P. Kruse, Y. Tang, S. Y. Jung, J. Qin, and W. Gu, "Negative regulation of the deacetylase SIRT1 by DBC1," Nature, vol. 451, no. 7178, pp. 587-590, 2008.

[10] R. Wang, Y. Zhang, J. Li, and C. Zhang, "Resveratrol ameliorates spatial learning memory impairment induced by $\mathrm{A} \beta 1-$ 42 in rats," Neuroscience, vol. 344, pp. 39-47, 2017.

[11] S. Michan, Y. Li, M. M.-H. Chou et al., "SIRT1 is essential for normal cognitive function and synaptic plasticity," The Journal of Neuroscience, vol. 30, no. 29, pp. 9695-9707, 2010.

[12] H. Yu, F. Zhang, and X. Guan, "Baicalin reverse depressive-like behaviors through regulation SIRT1-NF-kB signaling pathway in olfactory bulbectomized rats," Phytotherapy Research, vol. 33, no. 5, pp. 1480-1489, 2019.

[13] W.-J. Yan, D. B. Wang, D. Q. Ren et al., “AMPK $\alpha 1$ overexpression improves postoperative cognitive dysfunction in aged rats through AMPK-Sirt1 and autophagy signaling," Journal of Cellular Biochemistry, vol. 120, no. 7, pp. 11633-11641, 2019.

[14] J. Yan, A. Luo, J. Gao et al., "The role of SIRT1 in neuroinflammation and cognitive dysfunction in aged rats after anesthesia and surgery," American Journal of Translational Research, vol. 11, no. 3, pp. 1555-1568, 2019.

[15] F. M. Locatelli, T. Kawano, H. Iwata et al., "Resveratrol-loaded nanoemulsion prevents cognitive decline after abdominal surgery in aged rat," Journal of Pharmacological Sciences, vol. 137, no. 4, pp. 395-402, 2018.

[16] S.-W. Min, S. H. Cho, Y. Zhou et al., "Acetylation of tau inhibits its degradation and contributes to tauopathy," Neuron, vol. 67, no. 6, pp. 953-966, 2010.

[17] J. S. Hwang, E. S. Kang, S. G. Han et al., "Formononetin inhibits lipopolysaccharide-induced release of high mobility group box 1 by upregulating SIRT1 in a $\operatorname{PPAR} \delta$-dependent manner," PeerJ, vol. 6, article e4208, 2018.

[18] M. T. Lotze and K. J. Tracey, "High-mobility group box 1 protein (HMGB1): nuclear weapon in the immune arsenal," Nature Reviews Immunology, vol. 5, no. 4, pp. 331-342, 2005.

[19] T. Ravizza, G. Terrone, A. Salamone et al., "High mobility group box 1 is a novel pathogenic factor and a mechanistic biomarker for epilepsy," Brain, Behavior, and Immunity, vol. 72, pp. 14-21, 2018.

[20] X. Chen, S. Wu, C. Chen et al., "Omega-3 polyunsaturated fatty acid supplementation attenuates microglial-induced inflammation by inhibiting the HMGB1/TLR4/NF- $\kappa$ B pathway following experimental traumatic brain injury," Journal of Neuroinflammation, vol. 14, no. 1, p. 143, 2017.

[21] K. Sowndhararajan, P. Deepa, M. Kim, S. Park, and S. Kim, "Neuroprotective and cognitive enhancement potentials of Baicalin: a review," Brain Sciences, vol. 8, no. 6, p. 104, 2018.

[22] L. Zhang, D. Xing, W. Wang, R. Wang, and L. du, "Kinetic difference of baicalin in rat blood and cerebral nuclei after intravenous administration of Scutellariae Radix extract," Journal of Ethnopharmacology, vol. 103, no. 1, pp. 120-125, 2006.

[23] J. Fang, Y. Zhu, H. Wang et al., "Baicalin protects mice brain from apoptosis in traumatic brain injury model through activation of autophagy," Frontiers in Neuroscience, vol. 12, no. 1006, 2019.

[24] X. Shi, Y. Fu, S. S. Zhang, H. Ding, and J. Chen, "Baicalin attenuates subarachnoid hemorrhagic brain injury by modulating blood-brain barrier disruption, inflammation, and oxidative damage in mice," Oxidative Medicine and Cellular Longevity, vol. 2017, Article ID 1401790, 9 pages, 2017.

[25] Y. Cao, G. Li, Y. F. Wang et al., "Neuroprotective effect of baicalin on compression spinal cord injury in rats," Brain Research, vol. 1357, pp. 115-123, 2010.

[26] Y. Lu, G. Sun, F. Yang et al., "Baicalin regulates depression behavior in mice exposed to chronic mild stress via the Rac/LIMK/cofilin pathway," Biomedicine \& Pharmacotherapy, vol. 116, p. 109054, 2019.

[27] H.-Y. Chen, M. Geng, Y. Z. Hu, and J. H. Wang, "Effects of baicalin against oxidative stress injury of SH-SY5Y cells by upregulating SIRT1," Yao xue xue bao = Acta pharmaceutica Sinica, vol. 46, no. 9, pp. 1039-1044, 2011.

[28] L. Liu, Y. Dong, X. Shan, L. Li, B. Xia, and H. Wang, "Antidepressive effectiveness of baicalin in vitro and in vivo," Molecules, vol. 24, no. 2, p. 326, 2019.

[29] M.-A. SHAH, D.-J. PARK, J.-B. KANG, M.-O. KIM, and P.O. $\mathrm{KOH}$, "Baicalin attenuates lipopolysaccharide-induced neuroinflammation in cerebral cortex of mice via inhibiting nuclear factor kappa B (NF- $\kappa$ B) activation," Journal of Veterinary Medical Science, vol. 81, no. 9, pp. 1359-1367, 2019.

[30] L.-T. Guo, S. Q. Wang, J. Su et al., "Baicalin ameliorates neuroinflammation-induced depressive-like behavior through inhibition of toll-like receptor 4 expression via the PI3K/AKT/FoxO1 pathway," Journal of Neuroinflammation, vol. 16, no. 1, p. 95, 2019.

[31] M. H. Kan, T. Yang, H. Q. Fu et al., "Pyrrolidine dithiocarbamate prevents neuroinflammation and cognitive dysfunction after endotoxemia in rats," Frontiers in Aging Neuroscience, vol. 8, no. 175, 2016.

[32] H. Q. Fu, T. Yang, W. Xiao et al., "Prolonged neuroinflammation after lipopolysaccharide exposure in aged rats," PLoS One, vol. 9, no. 8, article e106331, 2014.

[33] X. Jin, M. Y. Liu, D. F. Zhang et al., "Baicalin mitigates cognitive impairment and protects neurons from microgliamediated neuroinflammation via suppressing NLRP3 inflammasomes and TLR4/NF- $\kappa \mathrm{B}$ signaling pathway," CNS Neuroscience \& Therapeutics, vol. 25, no. 5, pp. 575-590, 2019.

[34] L. Yu, Y. Sun, L. Cheng et al., "Melatonin receptor-mediated protection against myocardial ischemia/reperfusion injury: role of SIRT1," Journal of Pineal Research, vol. 57, no. 2, pp. 228-238, 2014.

[35] C. V. Vorhees and M. T. Williams, "Morris water maze: procedures for assessing spatial and related forms of learning and memory," Nature Protocols, vol. 1, no. 2, pp. 848-858, 2006.

[36] J. S. Hwang, W. J. Lee, E. S. Kang et al., "Ligand-activated peroxisome proliferator-activated receptor- $\delta$ and $-\gamma$ inhibit lipopolysaccharide-primed release of high mobility group box 1 through upregulation of SIRT1," Cell Death \& Disease, vol. 5, no. 10, article e1432, 2014.

[37] S.-W. Min, P. D. Sohn, Y. Li et al., "SIRT1 deacetylates tau and reduces pathogenic tau spread in a mouse model of tauopathy," The Journal of neuroscience : the official journal of the Society for Neuroscience, vol. 38, no. 15, pp. 3680-3688, 2018.

[38] N. Terrando, T. Yang, X. Wang et al., "Systemic HMGB1 neutralization prevents postoperative neurocognitive dysfunction in aged rats," Frontiers in Immunology, vol. 7, p. 441, 2016.

[39] Y. M. Kim, E. J. Park, H. J. Kim, and K. C. Chang, "Sirt1 Snitrosylation induces acetylation of HMGB1 in LPS-activated RAW264.7 cells and endotoxemic mice," Biochemical and 
Biophysical Research Communications, vol. 501, no. 1, pp. 7379, 2018.

[40] Z. Qi, Y. Zhang, S. Qi et al., "Salidroside inhibits HMGB1 acetylation and release through upregulation of SirT1 during inflammation," Oxidative Medicine and Cellular Longevity, vol. 2017, Article ID 9821543, 11 pages, 2017.

[41] X. Chen, C. Chen, S. Fan et al., "Omega-3 polyunsaturated fatty acid attenuates the inflammatory response by modulating microglia polarization through SIRT1-mediated deacetylation of the HMGB1/NF- $\kappa$ B pathway following experimental traumatic brain injury," Journal of Neuroinflammation, vol. 15, no. 1, p. 116, 2018.

[42] W. A. Banks, A. M. Gray, M. A. Erickson et al., "Lipopolysaccharide-induced blood-brain barrier disruption: roles of cyclooxygenase, oxidative stress, neuroinflammation, and elements of the neurovascular unit," Journal of Neuroinflammation, vol. 12, no. 1, p. 223, 2015.

[43] H. Li, P. Wang, F. Huang et al., "Astragaloside IV protects blood-brain barrier integrity from LPS-induced disruption via activating Nrf2 antioxidant signaling pathway in mice," Toxicology and Applied Pharmacology, vol. 340, pp. 58-66, 2018.

[44] S. A. Liddelow, K. A. Guttenplan, L. E. Clarke et al., "Neurotoxic reactive astrocytes are induced by activated microglia," Nature, vol. 541, no. 7638, pp. 481-487, 2017.

[45] E. Colombo and C. Farina, "Astrocytes: key regulators of neuroinflammation," Trends in Immunology, vol. 37, no. 9, pp. 608-620, 2016.

[46] H. Kumar, S. V. More, S. D. Han, J. Y. Choi, and D. K. Choi, "Promising therapeutics with natural bioactive compounds for improving learning and memory-a review of randomized trials," Molecules, vol. 17, no. 9, pp. 10503-10539, 2012.

[47] B. Dinda, S. Dinda, S. DasSharma, R. Banik, A. Chakraborty, and M. Dinda, "Therapeutic potentials of baicalin and its aglycone, baicalein against inflammatory disorders," European Journal of Medicinal Chemistry, vol. 131, pp. 68-80, 2017.

[48] X. Tang, Y. Zhao, Z. Zhou et al., "Resveratrol mitigates sevoflurane-induced neurotoxicity by the SIRT1-dependent regulation of $\mathrm{BDNF}$ expression in developing mice," Oxidative Medicine and Cellular Longevity, vol. 2020, Article ID 9018624, 18 pages, 2020.

[49] S. Pan, Y. Wu, L. Pei et al., "BML-111 reduces neuroinflammation and cognitive impairment in mice with sepsis via the SIRT1/NF- $\kappa$ B signaling pathway," Frontiers in Cellular Neuroscience, vol. 12, no. 267, 2018.

[50] H. Hong-qiang, S. Mang-qiao, X. Fen et al., "Sirt1 mediates improvement of isoflurane-induced memory impairment following hyperbaric oxygen preconditioning in middle-aged mice," Physiology \& Behavior, vol. 195, pp. 1-8, 2018.

[51] Y. Zhang, S. Anoopkumar-Dukie, D. Arora, and A. K. Davey, "Review of the anti-inflammatory effect of SIRT1 and SIRT2 modulators on neurodegenerative diseases," European Journal of Pharmacology, vol. 867, article 172847, 2020.

[52] J. Huang, W. Liu, D. M. Doycheva et al., "Ghrelin attenuates oxidative stress and neuronal apoptosis via GHSR$1 \alpha /$ AMPK/Sirt1/PGC-1 $\alpha / \mathrm{UCP} 2$ pathway in a rat model of neonatal HIE," Free Radical Biology and Medicine, vol. 141, pp. 322-337, 2019.

[53] S. Li, X. Sun, L. Xu et al., "Baicalin attenuates in vivo and in vitro hyperglycemia-exacerbated ischemia/reperfusion injury by regulating mitochondrial function in a manner dependent on AMPK," European Journal of Pharmacology, vol. 815, pp. 118-126, 2017.

[54] M. M. Rabadi, S. Xavier, R. Vasko, K. Kaur, M. S. Goligorksy, and B. B. Ratliff, "High-mobility group box 1 is a novel deacetylation target of Sirtuin1," Kidney International, vol. 87, no. 1, pp. 95-108, 2015.

[55] S. Wei, Y. Gao, X. Dai et al., "SIRT1-mediated HMGB1 deacetylation suppresses sepsis-associated acute kidney injury," American Journal of Physiology-Renal Physiology, vol. 316, no. 1, pp. F20-F31, 2019. 